\title{
SUFFICIENT OPTIMALITY CONDITIONS IN BILEVEL PROGRAMMING
}

\author{
Patrick Mehlitz, Alain B. Zemkoho ${ }^{\dagger}$
}

\begin{abstract}
This paper is concerned with the derivation of first- and second-order sufficient optimality conditions for optimistic bilevel optimization problems involving smooth functions. First-order sufficient optimality conditions are obtained by estimating the tangent cone to the feasible set of the bilevel program in terms of initial problem data. This is done by exploiting several different reformulations of the hierarchical model as a single-level problem. To obtain second-order sufficient optimality conditions, we exploit the so-called value function reformulation of the bilevel optimization problem, which is then tackled with the aid of second-order directional derivatives. The resulting conditions can be stated in terms of initial problem data in several interesting situations comprising the settings where the lower level is linear or possesses strongly stable solutions.
\end{abstract}

Keywords: bilevel optimization, first-order sufficient optimality conditions, second-order directional derivatives, second-order sufficient optimality conditions

$\operatorname{MSC}(2010): 90 C_{30}, 90 C_{33}, 90 C_{4} 6$

\section{INTRODUCTION}

Bilevel programming is without any doubt one of the most popular research areas in mathematical optimization. On the one hand, this interest is a consequence of numerous underlying real-world applications from a wide range of areas, including economics, finance, logistics, and chemistry, which can be modeled as hierarchical programs with two decision makers. On the other hand, bilevel programs are mathematically challenging since they are inherently nonsmooth, nonconvex, and irregular in the sense that a reformulation of the hierarchical model as single-level program results in surrogate problems which suffer from an inherent lack of smoothness, convexity, and regularity. A detailed introduction to the topic of bilevel programming can be found in the monographs [3, 12, 15, 44].

Despite the vast amount of work done so far on bilevel optimization, very little appears to have been done on sufficient optimality conditions for this problem class. The aim of this paper is to make new contributions in this area. Note that as in standard nonlinear optimization, such results have the potential to help accelerate work on numerical methods and stability analysis of bilevel programs. To proceed, we consider the standard bilevel optimization problem

$$
\min _{x, y}\{F(x, y) \mid G(x, y) \leq 0, y \in S(x)\}
$$

also known as the upper level problem. Here, the set-valued mapping $S: \mathbb{R}^{n} \rightrightarrows \mathbb{R}^{m}$ represents the optimal solution mapping of the so-called lower level problem and is defined by

$$
\forall x \in \mathbb{R}^{n}: \quad S(x):=\underset{y}{\operatorname{argmin}}\{f(x, y) \mid g(x, y) \leq 0\} .
$$

\footnotetext{
* Institute of Mathematics, Chair of Optimal Control. Brandenburgische Technische Universität Cottbus-Senftenberg, Germany.mehlitz@b-tu.de

† School of Mathematics. University of Southampton, United Kingdom, a.b.zemkoho@soton.ac.uk
} 
The functions $F, f: \mathbb{R}^{n} \times \mathbb{R}^{m} \rightarrow \mathbb{R}, G: \mathbb{R}^{n} \times \mathbb{R}^{m} \rightarrow \mathbb{R}^{p}$, and $g: \mathbb{R}^{n} \times \mathbb{R}^{m} \rightarrow \mathbb{R}^{q}$ are assumed to be twice continuously differentiable. Throughout the manuscript, the component maps of $G$ and $g$ will be denoted by $G_{1}, \ldots, G_{p}: \mathbb{R}^{n} \times \mathbb{R}^{m} \rightarrow \mathbb{R}$ and $g_{1}, \ldots, g_{q}: \mathbb{R}^{n} \times \mathbb{R}^{m} \rightarrow \mathbb{R}$, respectively. Recall that problem (BPP) has a two-level structure with $F$ (resp. $f$ ) denoting the upper level (resp. lower level) objective function and $G$ (resp. $g$ ) representing the upper level (resp. lower level) constraint functions. Observing that the minimization in (BPP) is carried out w.r.t. $x$ and $y$, the given model is closely related to the optimistic approach of the bilevel programming problem, see [49, Section 5.3] for details.

During the last decades, many papers appeared where necessary optimality conditions for (BPP) are derived via different single-level reformulations of the model. However, there is a significant gap in the literature on sufficient optimality conditions for (BPP). In [11, Theorem 4.2], the author presents a sufficient optimality condition for bilevel programming problems with uniquely determined lower level optimal solutions. The underlying analysis is based on conditions which ensure that the lower level optimal solution operator is singleton-valued and directionally differentiable. As demonstrated in [48, Theorem 6.1], exploiting standard second-order theory from smooth nonlinear programming, it is possible to infer sufficient optimality conditions for bilevel programming problems where upper and lower level problem are not constrained. In [14], the authors derive second-order optimality conditions for bilevel optimization problems using approximate derivatives. However, these optimality conditions are not stated in terms of initial problem data and hence, for large parts, do not reflect the inherent difficulties of bilevel optimization. A derivative-free sufficient optimality condition for bilevel optimization problems which is based on a max-min-equality is presented in [1]. In [20], the authors exploit generalized convexity assumptions and a new reformulation of the bilevel programming problem based on a so-called $\Psi$-function in order to infer a first-order sufficient optimality condition. Again, we emphasize that the obtained condition is not stated in terms of initial problem data.

In $[4,5,30]$, second-order sufficient optimality conditions for standard nonlinear programs have been derived which are based on the notion of a suitable critical cone and the second-order derivative of the underlying Lagrangian. Supposing that the feasible set of the program under consideration is given in an abstract way (e.g. by generalized inequalities), a generalization of these results is possible using the theory of suitable second-order tangent sets, see $[7,8,9,31,32,34]$. Let us mention that using generalized second-order derivatives, it is possible to derive second-order sufficient conditions in situations where nonsmooth optimization problems are under consideration, see [6, 8, 38, 39, 45].

In this paper, we are going to derive new first- and second-order sufficient optimality conditions for (BPP) in terms of initial problem data. For the first-order sufficient conditions, we exploit several approaches in order to approximate the tangent cone to the feasible set of (BPP) from above. The resulting primal conditions generally require that a certain system of equations does not possess a nontrivial solution. In order to obtain second-order sufficient optimality conditions for (BPP), we suggest to exploit the so-called value function reformulation of (BPP), see Section 3, as well as secondorder directional derivatives of all the involved functions. Our analysis is based on related investigations for standard nonlinear problems, see, e.g., [6, 41, 42], and semi-infinite nonlinear problems, see [39]. Throughout the paper, we present several examples in order to visualize the applicability of the obtained theory.

The remaining parts of this manuscript are organized as follows: In Section 2, we first introduce the basic notation used throughout the paper. Afterwards, some fundamentals of variational analysis are recalled. Furthermore, we state all notions of first- and second-order directional differentiability that we are going to exploit in this paper. Finally, we briefly summarize some essentials of constrained programming. Section 3 recalls some fundamental concepts from bilevel optimization. In Section 4 , first-order sufficient optimality conditions for (BPP) are derived. Therefore, we estimate the tangent cone to the feasible set of (BPP) from above using different single-level surrogate problems associated with (BPP), namely the value function reformulation, the generalized equation reformulation, and the Karush-Kuhn-Tucker (KKT) reformulation. In Section 5, second-order sufficient optimality conditions 
for (BPP) will be derived. Therefore, we exploit the value function reformulation. In order to describe the curvature of the optimal value function associated with (1.1), we make use of its second-order directional derivative. We first obtain an abstract result comprising some generalized derivatives of the optimal value function. In three exemplary settings, namely where the parametric optimization problem in (1.1) is fully linear, linear in $y$ and the parameters $x$ enters the lower level objective linearly, and where the lower level optimal solutions are strongly stable, the result is specified in terms of initial problem data. Finally, we close the paper in Section 6 with some concluding remarks comprising directions for future research.

\section{NOTATION AND PRELIMINARIES}

\subsection{BASIC NOTATION}

For a matrix $A \in \mathbb{R}^{m \times n}$ and a set $C \subseteq \mathbb{R}^{n}$, let us set $A C:=\{A x \mid x \in C\}$. Furthermore, we define by $\operatorname{dist}(x, C):=\inf \left\{\|x-y\|_{2} \mid y \in C\right\}$ the distance between $x$ and $C$. Here, $\|\cdot\|_{2}$ represents the Euclidean norm in $\mathbb{R}^{n}$. Forthwith, $\operatorname{conv} C, \operatorname{cl} C$, and int $C$ denote the convex hull, the closure, and the interior of $C$, respectively. We use $I_{n} \in \mathbb{R}^{n \times n}$ to express the identity. By dom $\theta:=\left\{x \in \mathbb{R}^{n}|| \theta(x) \mid<\infty\right\}$ and epi $\theta:=\left\{(x, \alpha) \in \mathbb{R}^{n} \times \mathbb{R} \mid \theta(x) \leq \alpha\right\}$, we denote the domain and the epigraph of a function $\theta: \mathbb{R}^{n} \rightarrow \overline{\mathbb{R}}$, respectively, where $\overline{\mathbb{R}}$ represents the extended real line. Similarly, for a set-valued mapping $\Theta: \mathbb{R}^{n} \rightrightarrows \mathbb{R}^{m}$, we use $\operatorname{dom} \Theta:=\left\{x \in \mathbb{R}^{n} \mid \Theta(x) \neq \varnothing\right\}$ and $\operatorname{gph} \Theta:=\left\{(x, y) \in \mathbb{R}^{n} \times \mathbb{R}^{m} \mid y \in \Theta(x)\right\}$ in order to represent the domain and the graph of $\Theta$, respectively. At $\bar{x} \in \operatorname{dom} \Theta, \Theta$ is said to be locally bounded whenever there exist a neighborhood $U \subseteq \mathbb{R}^{n}$ of $\bar{x}$ and a bounded set $B \subseteq \mathbb{R}^{m}$ such that $\Theta(x) \subseteq B$ holds for all $x \in U$. Furthermore, we call $\Theta$ metrically subregular at $(\bar{x}, \bar{y}) \in$ gph $\Theta$ whenever there are a neighborhood $U \subseteq \mathbb{R}^{n}$ of $\bar{x}$ and a constant $\kappa>0$ such that

$$
\forall x \in U: \quad \operatorname{dist}\left(x, \Theta^{-1}(\bar{y})\right) \leq \kappa \operatorname{dist}(\bar{y}, \Theta(x))
$$

is valid. Here, we used $\Theta^{-1}(\bar{y}):=\left\{x \in \mathbb{R}^{n} \mid \bar{y} \in \Theta(x)\right\}$ for the preimage of $\bar{y}$ under $\Theta$.

\subsection{VARIATIONAL ANALYSIS}

Let $C \subseteq \mathbb{R}^{n}$ be a nonempty set. Then, we call

$$
C^{\circ}:=\left\{y \in \mathbb{R}^{n} \mid \forall x \in C: x^{\top} y \leq 0\right\}
$$

the polar cone of $C$. Note that $C^{\circ}$ is a nonempty, closed, convex cone. For a nonempty index set $I$ and a family $\left\{C_{i}\right\}_{i \in I}$ of sets from $\mathbb{R}^{n}$, one easily obtains the polarization rule

$$
\left(\bigcup_{i \in I} C_{i}\right)^{\circ}=\bigcap_{i \in I} C_{i}^{\circ}
$$

by definition of the polar cone. For the polyhedral cone

$$
P:=\left\{x \in \mathbb{R}^{n} \mid A x \leq 0, B x=0\right\},
$$

where $A \in \mathbb{R}^{m_{1} \times n}$ and $B \in \mathbb{R}^{m_{2} \times n}$ are arbitrary matrices, one has (e.g., by Farkas' lemma) that

$$
P^{\circ}=\left\{A^{\top} \mu+B^{\top} v \in \mathbb{R}^{n} \mid \mu \in \mathbb{R}^{m_{1}}, \mu \geq 0, v \in \mathbb{R}^{m_{2}}\right\} .
$$

For a some point $\bar{x} \in C$, we refer to

$$
\mathcal{T}_{C}(\bar{x}):=\left\{d \in \mathbb{R}^{n} \mid \exists\left\{t_{k}\right\}_{k \in \mathbb{N}} \subseteq \mathbb{R} \exists\left\{d_{k}\right\}_{k \in \mathbb{N}} \subseteq \mathbb{R}^{n}: t_{k} \downarrow 0, d_{k} \rightarrow d, \bar{x}+t_{k} d_{k} \in C \forall k \in \mathbb{N}\right\}
$$


as the tangent (or Bouligand) cone to $C$ at $\bar{x}$. Furthermore,

$$
\widehat{\mathcal{N}}_{C}(\bar{x}):=\mathcal{T}_{C}(\bar{x})^{\circ}
$$

is referred to as the regular (or Fréchet) normal cone to $C$ at $\bar{x}$. The following lemma, which presents a calculus rule for tangents to linear images, is taken from [2, Proposition 4.3.9].

Lemma 2.1. Fix $C \subseteq \mathbb{R}^{n}$ and a matrix $A \in \mathbb{R}^{m \times n}$ as well as some point $\bar{x} \in C$ with

$$
\operatorname{ker} A \cap \mathcal{T}_{C}(\bar{x})=\{0\} .
$$

Then, it holds $\mathcal{T}_{A C}(A \bar{x})=\operatorname{cl}\left(A \mathcal{T}_{C}(\bar{x})\right)$. Above, $\operatorname{ker} A$ denotes the null space of $A$.

\subsection{GENERALIZED DIFFERENTIATION}

Throughout the section, we fix a function $\theta: \mathbb{R}^{n} \rightarrow \overline{\mathbb{R}}$ as well as some point $\bar{x} \in \operatorname{dom} \theta$. Let us first recall some fundamental notions of (first-order) directional differentiability, see, e.g., [10, 43]. For a direction $d \in \mathbb{R}^{n}$, the limits

$$
\theta^{+}(\bar{x} ; d):=\limsup _{t \downarrow 0} \frac{\theta(\bar{x}+t d)-\theta(\bar{x})}{t} \quad \text { and } \quad \theta^{-}(\bar{x} ; d):=\liminf _{t \downarrow 0} \frac{\theta(\bar{x}+t d)-\theta(\bar{x})}{t}
$$

are, respectively, referred to as the upper and lower Dini directional derivative of $\theta$ at $\bar{x}$ in direction $d$. In case of existence, we call

$$
\theta^{\prime}(\bar{x} ; d):=\lim _{t \downarrow 0} \frac{\theta(\bar{x}+t d)-\theta(\bar{x})}{t}
$$

the directional derivative (in Gâteaux's sense) of $\theta$ at $\bar{x}$ in direction $d$. Similarly, we introduce the Hadamard and Clarke directional derivative of $\theta$ at $\bar{x}$ in direction $d$, respectively, as

$$
\theta^{*}(\bar{x} ; d):=\lim _{t \downarrow 0, d^{\prime} \rightarrow d} \frac{\theta\left(\bar{x}+t d^{\prime}\right)-\theta(\bar{x})}{t} \quad \text { and } \quad \theta^{\circ}(\bar{x} ; d):=\limsup _{t \downarrow 0, x \rightarrow \bar{x}} \frac{\theta(x+t d)-\theta(x)}{t} .
$$

We call $\theta$ directionally differentiable (resp. Hadamard directionally differentiable, Clarke directionally differentiable) at $\bar{x}$ if the limit $\theta^{\prime}(\bar{x} ; d)\left(\right.$ resp. $\left.\theta^{*}(\bar{x} ; d), \theta^{\circ}(\bar{x} ; d)\right)$ exists for each $d \in \mathbb{R}^{n}$. Clearly, if $\theta$ is continuously differentiable at $\bar{x}$, then all these generalized derivatives coincide with $\nabla \theta(\bar{x})^{\top} d$ for each $d \in \mathbb{R}^{n}$. Let us note that whenever $\theta$ is locally Lipschitz continuous at $\bar{x}$, then it is Clarke directionally differentiable there. If, in addition to local Lipschitz continuity, $\theta$ is directionally differentiable at $\bar{x}$, then it is Hadamard directionally differentiable at $\bar{x}$ and $\theta^{\prime}(\bar{x} ; d)$ as well as $\theta^{*}(\bar{x} ; d)$ coincide. We call $\theta$ Clarke-regular at $\bar{x}$ if $\theta^{\prime}(\bar{x} ; d)=\theta^{\circ}(\bar{x} ; d)$ holds for all $d \in \mathbb{R}^{n}$. One can check that convex functions are Clarke regular at all points from int $\operatorname{dom} \theta$. Clearly, continuously differentiable functions are Clarke regular as well.

Supposing that $\theta$ is locally Lipschitz continuous at $\bar{x}$, its Clarke subdifferential at $\bar{x}$ given by

$$
\partial^{c} \theta(\bar{x}):=\left\{\xi \in \mathbb{R}^{n} \mid \forall d \in \mathbb{R}^{n}: \xi^{\top} d \leq \theta^{\circ}(\bar{x} ; d)\right\}
$$

is nonempty, convex, and compact. Let us note that this subdifferential construction enjoys full calculus, see [10], but is comparatively large w.r.t. set inclusion, i.e., it yields very weak necessary optimality conditions. Using the subdifferential, Clarke's directional derivative can be recovered by the formula

$$
\forall d \in \mathbb{R}^{n}: \quad \theta^{\circ}(\bar{x} ; d)=\max \left\{\xi^{\top} d \mid \xi \in \partial^{c} \theta(\bar{x})\right\} .
$$

Next, let $\theta$ be a convex function. Then, we call

$$
\partial \theta(\bar{x}):=\left\{\xi \in \mathbb{R}^{n} \mid \forall x \in \mathbb{R}^{n}: \theta(x) \geq \theta(\bar{x})+\xi^{\top}(x-\bar{x})\right\}
$$


the subdifferential of $\theta$ at $\bar{x}$ which is nonempty, closed, and convex. It is well known that

$$
\partial \theta(\bar{x})=\left\{\xi \in \mathbb{R}^{n} \mid \forall d \in \mathbb{R}^{n}: \xi^{\top} d \leq \theta^{\prime}(\bar{x} ; d)\right\}
$$

holds true. Thus, whenever a point $\bar{x}$ is under consideration where $\theta$ is locally Lipschitz continuous, then $\partial^{c} \theta(\bar{x})=\partial \theta(\bar{x})$ follows by Clarke regularity of $\theta$ at $\bar{x}$.

Below, we study a second-order directional derivative which has been exploited for the secondorder analysis of optimization problems in e.g. [6, 7, 8, 39, 41, 42]. For further information and some illustrative examples, we refer the interested reader to the aforementioned references. We say that $\theta$ is second-order directionally differentiable at $\bar{x}$ if the limit

$$
\theta^{\prime \prime}(\bar{x} ; d, w):=\lim _{t \downarrow 0} \frac{\theta\left(\bar{x}+t d+\frac{1}{2} t^{2} w\right)-\theta(\bar{x})-t \theta^{\prime}(\bar{x} ; d)}{\frac{1}{2} t^{2}}
$$

exists for each choice of $d, w \in \mathbb{R}^{n}$. In case of existence, the above limit is referred to as second-order directional derivative of $\theta$ at $\bar{x}$ w.r.t. the directions $d$ and $w$. Supposing that $\theta^{\prime \prime}(\bar{x} ; d, w)$ is finite, it holds $(\alpha \theta)^{\prime \prime}(\bar{x} ; d, w)=\alpha \theta^{\prime \prime}(\bar{x} ; d, w)$ for each $\alpha \in \mathbb{R}$. Furthermore, the sum rule is available for the second-order directional derivative provided all involved functions are second-order directionally differentiable. If $\theta$ is twice continuously differentiable at $\bar{x}$, then a second-order Taylor expansion yields

$$
\theta\left(\bar{x}+t d+\frac{1}{2} t^{2} w\right)=\theta(\bar{x})+\nabla \theta(\bar{x})^{\top} d+\frac{1}{2} t^{2}\left(\nabla \theta(\bar{x})^{\top} w+d^{\top} \nabla^{2} \theta(\bar{x}) d\right)+o\left(t^{2}\right)
$$

which shows that for all $d, w \in \mathbb{R}^{n}$, one has

$$
\theta^{\prime \prime}(\bar{x} ; d, w)=\nabla \theta(\bar{x})^{\top} w+d^{\top} \nabla^{2} \theta(\bar{x}) d .
$$

Subsequently, we state a calculus rule for the computation of second-order directional derivatives associated with the pointwise maximum of finitely many second-order directionally differentiable functions. It follows from the chain rule, see [8, Proposition 2.53], and can be found in the literature, see [6, Lemma 3.2] or [7, Section 4.1].

Lemma 2.2. Let $\theta_{1}, \ldots, \theta_{p}: \mathbb{R}^{n} \rightarrow \overline{\mathbb{R}}$ be Lipschitz continuous and second-order directionally differentiable at $\bar{x} \in \bigcap_{i=1}^{p} \operatorname{dom} \theta_{i}$. Furthermore, set $\theta:=\max \left\{\theta_{1}, \ldots, \theta_{p}\right\}$. Then, $\theta$ is second-order directionally differentiable at $\bar{x}$ and it holds

$$
\forall d \in \mathbb{R}^{n}: \quad \theta^{\prime}(\bar{x} ; d)=\max \left\{\theta_{i}^{\prime}(\bar{x} ; d) \mid i \in I(\bar{x})\right\}
$$

as well as

$$
\forall d, w \in \mathbb{R}^{n}: \quad \theta^{\prime \prime}(\bar{x} ; d, w)=\max \left\{\theta_{i}^{\prime \prime}(\bar{x} ; d, w) \mid i \in I(\bar{x}, d)\right\},
$$

where we use

$$
I(\bar{x}):=\left\{i \in\{1, \ldots, p\} \mid \theta(\bar{x})=\theta_{i}(\bar{x})\right\} \quad \text { and } \quad I(\bar{x}, d):=\left\{i \in I(\bar{x}) \mid \theta^{\prime}(\bar{x} ; d)=\theta_{i}^{\prime}(\bar{x} ; d)\right\} .
$$

In order to study sufficient optimality conditions with the aid of the second-order directional derivative introduced above, the presence of an additional regularity condition is indispensable. Here, we rely on the concept of second-order epi-regularity which dates back to [7] and is studied in [8, Sections 3.3.4 and 3.3.5].

Definition 2.3. Let $\theta: \mathbb{R}^{n} \rightarrow \overline{\mathbb{R}}$ be a given function and fix $\bar{x} \in \operatorname{dom} \theta$ where $\theta$ is second-order directionally differentiable. Then, $\theta$ is said to be second-order epi-regular at $\bar{x}$ in direction $d \in \mathbb{R}^{n}$ if for each path $w: \mathbb{R}_{+} \rightarrow \mathbb{R}^{n}$ which satisfies $t w(t) \rightarrow 0$ as $t \downarrow 0$, we have

$$
\theta\left(\bar{x}+t d+\frac{1}{2} t^{2} w(t)\right) \geq \theta(\bar{x})+t \theta^{\prime}(\bar{x} ; d)+\frac{1}{2} t^{2} \theta^{\prime \prime}(\bar{x} ; d, w(t))+o\left(t^{2}\right) .
$$

We say that $\theta$ is second-order epi-regular at $\bar{x}$ if it is second-order epi-regular at $\bar{x}$ in each direction from $\mathbb{R}^{n}$. 
Due to (2.1) and (2.2), each twice continuously differentiable functions is second-order epi-regular at each point in each direction. By definition, the sum of two second-order epi-regular functions is secondorder epi-regular as well. Furthermore, we note that a function $\theta: \mathbb{R}^{n} \rightarrow \overline{\mathbb{R}}$, which is second-order directionally differentiable and locally Lipschitz continuous at $\bar{x} \in \operatorname{dom} \theta$, is second-order epi-regular at $\bar{x}$ in direction $d \in \mathbb{R}^{n}$ if and only if the set epi $\theta$ is outer second-order regular at $(\bar{x}, \theta(\bar{x}))$, see [8, Definition 3.85, Proposition 3.95], and the latter particularly holds whenever epi $\theta$ is a polyhedron.

Lemma 2.4. Let $\theta_{1}, \ldots, \theta_{p}: \mathbb{R}^{n} \rightarrow \overline{\mathbb{R}}$ be Lipschitz continuous, second-order directionally differentiable, and second-order epi-regular at $\bar{x} \in \bigcap_{i=1}^{p} \operatorname{dom} \theta_{i}$. Furthermore, set $\theta:=\max \left\{\theta_{1}, \ldots, \theta_{p}\right\}$. Then, $\theta$ is second-order epi-regular at $\bar{x}$ as well.

Proof. Fix an arbitrary direction $d \in \mathbb{R}^{n}$ and an arbitrary path $w: \mathbb{R}_{+} \rightarrow \mathbb{R}^{n}$ satisfying $t w(t) \rightarrow 0$ as $t \downarrow 0$. For each $t \in \mathbb{R}_{+}$, there is an active index $i_{0}(t) \in\{1, \ldots, p\}$ which satisfies

$$
\theta(\bar{x})=\theta_{i_{0}(t)}(\bar{x}), \quad \theta^{\prime}(\bar{x} ; d)=\theta_{i_{0}(t)}^{\prime}(\bar{x} ; d), \quad \theta^{\prime \prime}(\bar{x} ; d, w(t))=\theta_{i_{0}(t)}^{\prime \prime}(\bar{x} ; d, w(t)),
$$

see Lemma 2.2. Noting that all the functions $\theta_{i}$ are second-order epi-regular at $\bar{x}$ in direction $d$, it holds

$$
\theta_{i}\left(\bar{x}+t d+\frac{1}{2} t^{2} w(t)\right) \geq \theta_{i}(\bar{x})+t \theta_{i}^{\prime}(\bar{x} ; d)+\frac{1}{2} t^{2} \theta_{i}^{\prime \prime}(\bar{x} ; d, w(t))+o_{i}\left(t^{2}\right)
$$

for all $i=1, \ldots, p$. For each $t \in \mathbb{R}_{+}$, we set $o(t):=\min \left\{o_{i}(t) \mid i \in\{1, \ldots, p\}\right\}$. Then, we have

$$
\begin{aligned}
\theta\left(\bar{x}+t d+\frac{1}{2} t^{2} w(t)\right) & \geq \theta_{i_{0}(t)}\left(\bar{x}+t d+\frac{1}{2} t^{2} w(t)\right) \\
& \geq \theta_{i_{0}(t)}(\bar{x})+t \theta_{i_{0}(t)}^{\prime}(\bar{x} ; d)+\frac{1}{2} t^{2} \theta_{i_{0}(t)}^{\prime \prime}(\bar{x} ; d, w(t))+o\left(t^{2}\right) \\
& =\theta(\bar{x})+t \theta^{\prime}(\bar{x} ; d)+\frac{1}{2} t^{2} \theta^{\prime \prime}(\bar{x} ; d, w(t))+o\left(t^{2}\right) .
\end{aligned}
$$

Since $d$ was chosen arbitrarily, the desired result follows.

\subsection{PRELIMINARIES ON CONSTRAINED PROGRAMMING}

In this section, we review some optimality conditions for the general optimization problem

$$
\min \{\theta(x) \mid x \in X\}
$$

where $\theta: \mathbb{R}^{n} \rightarrow \overline{\mathbb{R}}$ is a given functional and $X \subseteq \mathbb{R}^{n}$ is a nonempty, closed set. First of all, the following first-order necessary optimality condition can be easily proven using standard arguments.

Lemma 2.5. Let $\bar{x} \in \operatorname{dom} \theta$ be a local minimizer of $(\mathrm{P})$, where $\theta$ is Hadamard directionally differentiable. Then, it holds that

$$
\forall d \in \mathcal{T}_{X}(\bar{x}): \quad \theta^{*}(\bar{x} ; d) \geq 0 .
$$

Next, we want to deal with sufficient optimality conditions which address $(\mathrm{P})$. To proceed, we use the notion of growth conditions.

Definition 2.6. Fix $\bar{x} \in X$. We say that the growth condition of order $\alpha>0$ holds at $\bar{x}$ for (P) whenever there are a neighborhood $U \subseteq \mathbb{R}^{n}$ of $\bar{x}$ and a constant $C>0$ such that the following is true:

$$
\forall x \in X \cap U: \quad \theta(x) \geq \theta(\bar{x})+C\|x-\bar{x}\|_{2}^{\alpha} .
$$

Clearly, whenever the growth condition of order $\alpha>0$ holds at some point $\bar{x} \in X$ for $(\mathrm{P})$, then $\bar{x}$ is a strict local minimizer of $(\mathrm{P})$. A sufficient optimality condition for $(\mathrm{P})$ is said to be of order $\alpha>0$, if it implies validity of the growth condition of order $\alpha$ for $(\mathrm{P})$ at the reference point. Sufficient optimality conditions from the literature are of order 1 or 2 in most of the cases.

Below, we first study a rather general first-order sufficient optimality condition for $(\mathrm{P})$. The proof, although it is folklore, is included for the reader's convenience. 
Proposition 2.7. Let $\bar{x} \in \operatorname{dom} \theta$ be a feasible point of $(\mathrm{P})$, where $\theta$ is Hadamard directionally differentiable and assume that

$$
\forall d \in \mathcal{T}_{X}(\bar{x}) \backslash\{0\}: \quad \theta^{*}(\bar{x} ; d)>0 .
$$

Then, the first-order growth condition holds at $\bar{x}$ for $(\mathrm{P})$. Particularly, $\bar{x}$ is a strict local minimizer of $(\mathrm{P})$.

Proof. Assume on the contrary that the first-order growth condition does not hold at $\bar{x}$ for (P). Then, we find a sequence $\left\{x_{k}\right\}_{k \in \mathbb{N}} \subseteq X$ converging to $\bar{x}$ such that

$$
\forall k \in \mathbb{N}: \quad \theta\left(x_{k}\right)<\theta(\bar{x})+\frac{1}{k}\left\|x_{k}-\bar{x}\right\|_{2}
$$

holds true. We set $t_{k}:=\left\|x_{k}-\bar{x}\right\|_{2}$ and $d_{k}:=\left(x_{k}-\bar{x}\right) / t_{k}$ for each $k \in \mathbb{N}$ and observe $t_{k} \downarrow 0$. Furthermore, $\left\{d_{k}\right\}_{k \in \mathbb{N}}$ is bounded and converges w.l.o.g. to some $d \in \mathcal{T}_{X}(\bar{x}) \backslash\{0\}$. By definition of Hadamard's directional derivative, we obtain

$$
\theta^{*}(\bar{x} ; d)=\lim _{k \rightarrow \infty} \frac{\theta\left(\bar{x}+t_{k} d_{k}\right)-\theta(\bar{x})}{t_{k}}=\lim _{k \rightarrow \infty} \frac{\theta\left(x_{k}\right)-\theta(\bar{x})}{t_{k}} \leq \lim _{k \rightarrow \infty} \frac{\left\|x_{k}-\bar{x}\right\|_{2}}{k t_{k}}=0
$$

which contradicts the proposition's assumptions.

Observe that the necessary and sufficient first-order optimality conditions from Lemma 2.5 and Proposition 2.7, respectively, only differ w.r.t. the appearing relation the Hadamard directional derivative has to satisfy for nonvanishing tangent directions to $X$ at the reference point. That is why we speak of a pair of no-gap first-order optimality conditions.

In numerous models of mathematical optimization, the feasible set $X$ is described via inequality constraints. Thus, let us assume for a moment that $X$ is given by

$$
X:=\left\{x \in \mathbb{R}^{n} \mid \forall i \in\{1, \ldots, m\}: \theta_{i}(x) \leq 0\right\}
$$

where $\theta_{1}, \ldots, \theta_{m}: \mathbb{R}^{n} \rightarrow \overline{\mathbb{R}}$ are given functionals. In order to apply the sufficient optimality condition from Proposition 2.7 efficiently to this particular setting, one needs a computable upper approximate of the tangent cone. It is a standard idea in mathematical programming to exploit a linearization of the constraining functionals $\theta_{1}, \ldots, \theta_{m}$ for that purpose. Let us fix a point $\bar{x} \in X$ where the functionals $\theta_{1}, \ldots, \theta_{m}$ are locally Lipschitz continuous. We call

$$
\mathcal{L}_{X}(\bar{x}):=\left\{d \in \mathbb{R}^{n} \mid \theta_{i}^{-}(\bar{x} ; d) \leq 0 \forall i \in I(\bar{x})\right\}
$$

the linearization cone to $X$ at $\bar{x}$. Above, we used $I(\bar{x}):=\left\{i \in\{1, \ldots, m\} \mid \theta_{i}(\bar{x})=0\right\}$. Observe that in case where the functionals $\theta_{i}, i \in I(\bar{x})$, are continuously differentiable at $\bar{x}$, then $\mathcal{L}_{X}(\bar{x})$ coincides with the classical linearization cone of standard nonlinear programming and is polyhedral. In the more general case discussed above, $\mathcal{T}_{X}(\bar{x})$ is only a closed cone which does not need to be convex since the lower Dini directional derivative is only positively homogeneous but not necessarily linear w.r.t. the direction. By standard arguments, we obtain that $\mathcal{L}_{X}(\bar{x})$ provides an upper estimate of $\mathcal{T}_{X}(\bar{x})$.

Lemma 2.8. Let $X$ be given as in (2.3). Fix $\bar{x} \in X$ and assume that $\theta_{1}, \ldots, \theta_{m}$ are locally Lipschitz continuous at $\bar{x}$. Then, we have $\mathcal{T}_{X}(\bar{x}) \subseteq \mathcal{L}_{X}(\bar{x})$.

Clearly, the converse inclusion $\mathcal{T}_{X}(\bar{x}) \supseteq \mathcal{L}_{X}(\bar{x})$ holds in general only under additional assumptions. However, equality would be beneficial in order to obtain a first-order sufficient optimality condition in Proposition 2.7 which is as weak as possible. Thinking about standard nonlinear programming, one might be tempted to say that Abadie's Constraint Qualification (ACQ) holds at $\bar{x}$ whenever it holds $\mathcal{T}_{X}(\bar{x})=\mathcal{L}_{X}(\bar{x})$. In case where the functions $\theta_{1}, \ldots, \theta_{m}$ are continuously differentiable, there exist several constraint qualifications stronger than ACQ which are stated in terms of initial problem data. In this manuscript, we refer to the Linear Independence Constraint Qualification (LICQ), the MangasarianFromovitz constraint qualification (MFCQ), and the Constant Rank Constraint Qualification (CRCQ) in some situations, see, e.g., $[4,28]$ for the definitions and some discussion.

Using Lemma 2.8, we obtain the following corollary from Proposition 2.7. 
Corollary 2.9. Let $\bar{x} \in \operatorname{dom} \theta$ be a feasible point of $(\mathrm{P})$ where $X$ is given as in (2.3). Furthermore, assume that the functionals $\theta_{1}, \ldots, \theta_{m}$ are locally Lipschitz continuous at $\bar{x}$. Finally, suppose that the system

$$
\begin{aligned}
\theta^{*}(\bar{x} ; d) & \leq 0 \\
\theta_{i}^{-}(\bar{x} ; d) & \leq 0 \quad i \in I(\bar{x})
\end{aligned}
$$

does not possess a nontrivial solution. Then, the first-order growth condition holds at $\bar{x}$ for $(\mathrm{P})$.

Below, we combine the concepts of second-order directional differentiability and second-order epiregularity in order to state a sufficient second-order optimality condition which addresses $(\mathrm{P})$ whenever $X:=\mathbb{R}^{n}$ holds. We note, however, that this statement can be extended to inequality-constrained mathematical problems and even to bilevel optimization as we will see in Section 5 . The result and its proof can be found in [39, Proposition 2.1].

Proposition 2.10. Set $X:=\mathbb{R}^{n}$. Fix $\bar{x} \in \operatorname{dom} \theta$ where $\theta$ is second-order directionally differentiable and second-order epi-regular. Furthermore, assume that

$$
\forall d \in \mathbb{R}^{n}: \quad \theta^{\prime}(\bar{x} ; d) \geq 0
$$

as well as

$$
\forall d \in\left\{r \in \mathbb{R}^{n} \mid \theta^{\prime}(\bar{x} ; r)=0\right\} \backslash\{0\}: \quad \inf _{w \in \mathbb{R}^{n}} \theta^{\prime \prime}(\bar{x} ; d, w)>0
$$

hold. Then, the second-order growth condition holds at $\bar{x}$ for $(\mathrm{P})$.

\section{PRELIMINARIES FROM BILEVEL OPTIMIZATION}

Let us provide some notation addressing (BPP). For later use, we introduce the lower level feasible set mapping $K: \mathbb{R}^{n} \rightrightarrows \mathbb{R}^{m}$ by

$$
\forall x \in \mathbb{R}^{n}: \quad K(x):=\left\{y \in \mathbb{R}^{m} \mid g(x, y) \leq 0\right\} .
$$

Let $M \subseteq \mathbb{R}^{n} \times \mathbb{R}^{m}$ denote the feasible set of problem (BPP) and $L: \mathbb{R}^{n} \times \mathbb{R}^{m} \times \mathbb{R}^{q} \rightarrow \mathbb{R}$ the lower level Lagrangian function defined by

$$
\forall(x, y, \lambda) \in \mathbb{R}^{n} \times \mathbb{R}^{m} \times \mathbb{R}^{q}: \quad L(x, y, \lambda):=f(x, y)+\lambda^{\top} g(x, y) .
$$

Using the latter notation, we can define the set of lower level Lagrange multipliers as

$$
\Lambda(x, y):=\left\{\lambda \in \mathbb{R}^{q} \mid \nabla_{y} L(x, y, \lambda)=0, \lambda \geq 0, \lambda^{\top} g(x, y)=0\right\}
$$

for points $(x, y) \in \operatorname{gph} K$. For a point $(\bar{x}, \bar{y}) \in \operatorname{gph} K$, we use

$$
\bar{I}^{g}=I^{g}(\bar{x}, \bar{y}):=\left\{i \in\{1, \ldots, q\} \mid g_{i}(\bar{x}, \bar{y})=0\right\}
$$

in order to represent the lower level index set of active constraints w.r.t. $(\bar{x}, \bar{y})$. If $(\bar{x}, \bar{y})$ satisfies $G(\bar{x}, \bar{y}) \leq 0$, the following definition is reasonable as well:

$$
\bar{I}^{G}=I^{G}(\bar{x}, \bar{y}):=\left\{i \in\{1, \ldots, p\} \mid G_{i}(\bar{x}, \bar{y})=0\right\} .
$$

The following lower level value function reformulation of problem (BPP) will be important for the developments in this paper:

$$
\min _{x, y}\{F(x, y) \mid G(x, y) \leq 0, f(x, y) \leq \varphi(x), g(x, y) \leq 0\} .
$$


This is a nonsmooth and nonconvex constrained optimization problem, given that the involved lower level value function $\varphi$ defined by

$$
\forall x \in \mathbb{R}^{n}: \quad \varphi(x):=\inf _{y}\{f(x, y) \mid g(x, y) \leq 0\}
$$

is typically nondifferentiable. Even when all the functions involved in (BPP) are fully convex, i.e., $f$ and $g$ are convex w.r.t. $(x, y)$, the feasible set of problem (LLVF) is still typically nonconvex. Furthermore, it is folklore that (LLVF) is irregular in the sense that standard constraint qualifications from nonsmooth programming do not hold at all feasible points of (LLVF). However, we note that (BPP) and (LLVF) are fully equivalent optimization problems.

We fix some point $(\bar{x}, \bar{y}) \in$ gph $K$. Generally, we say that a constraint qualification holds for the lower level problem $(1.1)$ at $(\bar{x}, \bar{y})$ if it is valid at the point $\bar{y}$ within the set $K(\bar{x})$ for fixed parameter. Exemplary, the lower level linear independence constraint qualification (LLICQ) will be said to hold at $(\bar{x}, \bar{y})$ if the family $\left\{\nabla_{y} g_{i}(\bar{x}, \bar{y})\right\}_{i \in \bar{I}^{g}}$ of gradients is linearly independent. Supposing that $(\bar{x}, \bar{y}) \in \operatorname{gph} S$ is given such that a constraint qualification holds for (1.1) at this point, the set $\Lambda(\bar{x}, \bar{y})$ is nonempty. For $\lambda \in \Lambda(\bar{x}, \bar{y})$, we introduce the lower level critical cone at $(\bar{x}, \bar{y})$ as stated below:

$$
C^{L}(\bar{x}, \bar{y}):=\left\{\begin{array}{l|l}
\delta_{y} \in \mathbb{R}^{m} & \begin{array}{l}
\nabla_{y} g_{i}(\bar{x}, \bar{y})^{\top} \delta_{y} \leq 0 \\
\nabla_{y} g_{i}(\bar{x}, \bar{y})^{\top} \delta_{y}=0
\end{array} \quad i \in \bar{I}^{g}, \lambda_{i}=0 \\
\lambda_{i}>0
\end{array}\right\} .
$$

It is well known that this definition actually does not depend on the particular choice of the multiplier since we also have

$$
C^{L}(\bar{x}, \bar{y})=\left\{\begin{array}{l|l}
\delta_{y} \in \mathbb{R}^{m} & \begin{array}{l}
\nabla_{y} f(\bar{x}, \bar{y})^{\top} \delta_{y}=0 \\
\nabla_{y} g_{i}(\bar{x}, \bar{y})^{\top} \delta_{y} \leq 0
\end{array} \quad i \in \bar{I}^{g}
\end{array}\right\}
$$

by elementary calculations exploiting the definition of $\Lambda(\bar{x}, \bar{y})$. Note that under validity of LLICQ, the set $\Lambda(\bar{x}, \bar{y})$ is a singleton. In this situation, lower level Strict Complementarity (LSC) is said to hold whenever $\bar{\lambda}_{j}>0$ is valid for all $j \in \bar{I}^{g}$ where $\bar{\lambda}$ is the unique element from $\Lambda(\bar{x}, \bar{y})$. Finally, the lower level Second-Order Sufficient Condition (LSOSC) is said to hold at $(\bar{x}, \bar{y})$ if we have

$$
\forall \delta_{y} \in C^{L}(\bar{x}, \bar{y}) \backslash\{0\} \quad \exists \lambda \in \Lambda(\bar{x}, \bar{y}): \quad \delta_{y}^{\top} \nabla_{y y}^{2} L(\bar{x}, \bar{y}, \lambda) \delta_{y}>0 .
$$

Next, we briefly recall a partial exact penalization principle addressing (LLVF) popular in bilevel programming. Therefore, we fix a local minimizer $(\bar{x}, \bar{y}) \in \mathbb{R}^{n} \times \mathbb{R}^{m}$ of (BPP). For a neighborhood $U \subseteq \mathbb{R}^{n} \times \mathbb{R}^{m} \times \mathbb{R}$ of $(\bar{x}, \bar{y}, 0)$ and define the set of locally feasible points of the problem resulting from a perturbation on the value function of problem (LLVF) by

$$
\mathcal{F}_{U}:=\{(x, y, \varsigma) \in U \mid G(x, y) \leq 0, f(x, y)-\varphi(x)+\varsigma=0, g(x, y) \leq 0\} .
$$

Problem (LLVF) will be said to be partially calm at $(\bar{x}, \bar{y})$ if there exist $\kappa>0$ and a neighborhood $U \subseteq \mathbb{R}^{n} \times \mathbb{R}^{m} \times \mathbb{R}$ of $(\bar{x}, \bar{y}, 0)$ such that

$$
\forall(x, y, \varsigma) \in \mathcal{F}_{U}: \quad F(x, y)-F(\bar{x}, \bar{y})+\kappa|\varsigma| \geq 0 .
$$

Partial calmness dates back to the seminal paper [48] where the authors show that (LLVF) is partially calm at one of its local minimizers $(\bar{x}, \bar{y})$ if and only if there is some $\bar{\kappa}>0$ such that $(\bar{x}, \bar{y})$ is also a local minimizer of the problem

$$
\min _{x, y}\{F(x, y)+\kappa(f(x, y)-\varphi(x)) \mid G(x, y) \leq 0, g(x, y) \leq 0\}
$$


for each $\kappa \geq \bar{\kappa}$. Clearly, dealing with (3.3) is beneficial since standard constraint qualifications may hold at the feasible points of this problem. As a consequence, KKT-type necessary optimality conditions, which exploit some generalized derivative of $\varphi$, can be used to characterize the local minimizer $(\bar{x}, \bar{y})$. Observe that the resulting KKT-system may be interpreted as the KKT-system of (LLVF) where the penalization parameter $\kappa$ plays the role of the Lagrange multiplier associated with the constraint $f(x, y)-\varphi(x) \leq 0$. In [17, Section 4], [33, Section 6], and [48], the authors present several conditions which ensure validity of partial calmness at local minimizers. Exemplary, let us mention that bilevel optimization problems with fully linear lower level are partially calm at all their local minimizers due to [48, Proposition 4.1]. In general, however, as highlighted in [27], this property is very restrictive.

\section{FIRST-ORDER SUFFICIENT OPTIMALITY CONDITIONS}

As already mentioned in Section 2.4, the term first-order refers to the fact that the optimality conditions derived in this section imply the fulfillment of the first-order growth condition at the reference point for (BPP). As we will see, depending on the exploited approach, the obtained sufficient optimality conditions may contain first- or second-order derivative information of the lower level program, i.e. we would like to make clear that a first-order sufficient optimality condition for (BPP) still may comprise derivative information of order higher than one.

From Proposition 2.7, we obtain the following general sufficient optimality condition which will be the base of our analysis in this section. Recall that the set $M$ denotes the feasible set of (BPP).

Lemma 4.1. Let $(\bar{x}, \bar{y}) \in \mathbb{R}^{n} \times \mathbb{R}^{m}$ be a feasible point of (BPP) such that the system

$$
\nabla F(\bar{x}, \bar{y})^{\top} d \leq 0 \quad d \in \mathcal{T}_{M}(\bar{x}, \bar{y})
$$

does not possess a nontrivial solution. Then, the first-order growth condition holds at $(\bar{x}, \bar{y})$ for (BPP). Particularly, $(\bar{x}, \bar{y})$ is a strict local minimizer of (BPP).

The above lemma is of limited practical use as long as no reasonable upper estimate of $\mathcal{T}_{M}(\bar{x}, \bar{y})$ in terms of initial problem data is available. Here, we are going to discuss such estimates.

\subsection{VALUE-FUNCTION APPROACH}

Using the optimal value function $\varphi, M$ possesses the equivalent representation

$$
M=\left\{(x, y) \in \mathbb{R}^{n} \times \mathbb{R}^{m} \mid G(x, y) \leq 0, f(x, y) \leq \varphi(x), g(x, y) \leq 0\right\} .
$$

Thus, the computation of an upper estimate of $\mathcal{T}_{M}(\bar{x}, \bar{y})$ can be carried with the aid of Lemma 2.8, and Corollary 2.9 yields a sufficient optimality condition for (BPP).

Theorem 4.2. Let $(\bar{x}, \bar{y}) \in \mathbb{R}^{n} \times \mathbb{R}^{m}$ be a feasible point of (BPP). Assume that $K$ is locally bounded at $\bar{x}$ while LMFCQ holds at all points $(\bar{x}, y) \in \operatorname{gph} S$. Furthermore, assume that the system

$$
\begin{aligned}
\nabla F(\bar{x}, \bar{y})^{\top} d & \leq 0 \\
\nabla G_{i}(\bar{x}, \bar{y})^{\top} d & \leq 0 \quad i \in \bar{I}^{G} \\
\nabla f(\bar{x}, \bar{y})^{\top} d-\varphi^{+}\left(\bar{x} ; \delta_{x}\right) & \leq 0 \\
\nabla g_{i}(\bar{x}, \bar{y})^{\top} d & \leq 0 \quad i \in \bar{I}^{g}
\end{aligned}
$$

does not possess a nontrivial solution $d:=\left(\delta_{x}, \delta_{y}\right) \in \mathbb{R}^{n} \times \mathbb{R}^{m}$. Then, $(\bar{x}, \bar{y})$ is a strict local minimizer of (BPP). 
Proof. The assumptions of the theorem guarantee that $\varphi$ is locally Lipschitz continuous at $\bar{x}$, see, e.g., [12, Theorem 4.17]. Thus, we can apply Corollary 2.9 to (LLVF). The result follows observing that

$$
(f-\varphi)^{-}((\bar{x}, \bar{y}) ; d)=\nabla f(\bar{x}, \bar{y})^{\top} d+(-\varphi)^{-}\left(\bar{x} ; \delta_{x}\right)=\nabla f(\bar{x}, \bar{y})^{\top} d-\varphi^{+}\left(\bar{x} ; \delta_{x}\right)
$$

holds by definition of the upper Dini directional derivative for all $d:=\left(\delta_{x}, \delta_{y}\right) \in \mathbb{R}^{n} \times \mathbb{R}^{m}$.

Example 4.3. Let us consider the bilevel programming problem

$$
\min _{x, y}\left\{\frac{1}{2}(x+3)^{2}+\frac{1}{2}(y+1)^{2} \mid y \in S(x)\right\}
$$

where $S: \mathbb{R} \rightrightarrows \mathbb{R}$ is the solution set mapping of $\min _{y}\{x y \mid 0 \leq y \leq 1\}$. Noting that the lower level feasible set is independent of $x$, compact, and regular, the associated optimal value function is locally Lipschitz continuous. Indeed, a simple calculation shows

$$
\forall x \in \mathbb{R}: \quad S(x)=\left\{\begin{array}{ll}
\{1\} & x<0, \\
{[0,1]} & x=0, \\
\{0\} & x>0,
\end{array} \quad \text { and } \quad \varphi(x)=\min \{x ; 0\} .\right.
$$

Let us consider the point $(\bar{x}, \bar{y}):=(0,0)$. The associated system $(4.1)$ reads as

$$
3 \delta_{x}+\delta_{y} \leq 0, \quad-\min \left\{\delta_{x} ; 0\right\} \leq 0, \quad-\delta_{y} \leq 0 .
$$

Clearly, this system possesses only the trivial solution $\left(\delta_{x}, \delta_{y}\right)=(0,0)$ which is why $(\bar{x}, \bar{y})$ is a strict local minimizer of the considered bilevel optimization problem.

Below, we present some corollaries of Theorem 4.2 where the abstract upper Dini derivative of $\varphi$ is approximated in terms of initial problem data. Under the assumptions of Theorem 4.2, the upper Dini directional derivative of $\varphi$ can be estimated from above, see [12, Theorem 4.15]. Using this approximate, the following corollary follows easily.

Corollary 4.4. Let $(\bar{x}, \bar{y}) \in \mathbb{R}^{n} \times \mathbb{R}^{m}$ be a feasible point of (BPP). Assume that $K$ is locally bounded at $\bar{x}$ while LMFCQ holds at all points $(\bar{x}, y) \in \mathrm{gph}$ S. Finally, assume that the system (4.1a), (4.1b), (4.1d), and

$$
\nabla_{y} f(\bar{x}, \bar{y})^{\top} \delta_{y}-\inf _{y \in S(\bar{x})} \max _{\lambda \in \Lambda(\bar{x}, y)}\left(\left(\nabla_{x} f(\bar{x}, y)-\nabla_{x} f(\bar{x}, \bar{y})\right)^{\top} \delta_{x}+\lambda^{\top} \nabla_{x} g(\bar{x}, y) \delta_{x}\right) \leq 0
$$

does not possess a nontrivial solution $d:=\left(\delta_{x}, \delta_{y}\right) \in \mathbb{R}^{n} \times \mathbb{R}^{m}$. Then, $(\bar{x}, \bar{y})$ is a strict local minimizer of (BPP).

In case where the lower level problem is convex w.r.t. $y$, i.e., if the lower level data functions $f$ and $g_{1}, \ldots, g_{q}$ are convex w.r.t. $y$ for each choice of the parameter $x$, the assumptions of Theorem 4.2 already guarantee that $\varphi$ is directionally differentiable at the reference point, see [12, Theorem 4.16]. This result already provides a ready-to-use formula for the directional derivative which yields a slightly better result than the one presented in Corollary 4.4 .

Corollary 4.5. Let $(\bar{x}, \bar{y}) \in \mathbb{R}^{n} \times \mathbb{R}^{m}$ be a feasible point of (BPP) where the lower level problem is convex w.r.t. $y$. Assume that $K$ is locally bounded at $\bar{x}$ while LMFCQ holds at all points $(\bar{x}, y) \in \operatorname{gph} S$. Finally, assume that the system (4.1a), (4.1b), (4.1d), and

$$
\nabla_{y} f(\bar{x}, \bar{y})^{\top} \delta_{y}-\min _{y \in S(\bar{x})} \max _{\lambda \in \Lambda(\bar{x}, y)}\left(\left(\nabla_{x} f(\bar{x}, y)-\nabla_{x} f(\bar{x}, \bar{y})\right)^{\top} \delta_{x}+\lambda^{\top} \nabla_{x} g(\bar{x}, y) \delta_{x}\right) \leq 0
$$

does not possess a nontrivial solution $d:=\left(\delta_{x}, \delta_{y}\right) \in \mathbb{R}^{n} \times \mathbb{R}^{m}$. Then, $(\bar{x}, \bar{y})$ is a strict local minimizer of (BPP). 
Finally, the situation of a fully convex lower level problem is even more comfortable since we do not need to consider the minimum over all lower level solutions associated with the reference point, see [12, Corollary 4.7]

Corollary 4.6. Let $(\bar{x}, \bar{y}) \in \mathbb{R}^{n} \times \mathbb{R}^{m}$ be a feasible point of (BPP) where the lower level problem is jointly convex w.r.t. $(x, y)$. Assume that $K$ is locally bounded at $\bar{x}$ while LMFCQ holds at all points $(\bar{x}, y) \in \operatorname{gph} S$. Finally, assume that the system (4.1a), (4.1b), (4.1d), and

$$
\nabla_{y} f(\bar{x}, \bar{y})^{\top} \delta_{y}-\max _{\lambda \in \Lambda(\bar{x}, \bar{y})} \lambda^{\top} \nabla_{x} g(\bar{x}, \bar{y}) \delta_{x} \leq 0
$$

does not possess a nontrivial solution $d:=\left(\delta_{x}, \delta_{y}\right) \in \mathbb{R}^{n} \times \mathbb{R}^{m}$. Then, $(\bar{x}, \bar{y})$ is a strict local minimizer of (BPP).

As soon as the negative value function $-\varphi$ is regular in Clarke's sense, the assertion of Theorem 4.2 can be stated in terms of Clarke's subdifferential of $\varphi$.

Theorem 4.7. Let $(\bar{x}, \bar{y}) \in \mathbb{R}^{n} \times \mathbb{R}^{m}$ be a feasible point of (BPP). Assume that $\varphi$ is locally Lipschitz continuous at $\bar{x}$ while $-\varphi$ is Clarke-regular at $\bar{x}$. Furthermore, suppose that the polar cone of the set

$$
Q:=\{\nabla F(\bar{x}, \bar{y})\} \cup\left\{\nabla G_{i}(\bar{x}, \bar{y}) \mid i \in \bar{I}^{G}\right\}
$$

$$
\cup\left\{\nabla f(\bar{x}, \bar{y})-\left[\begin{array}{l}
\xi \\
0
\end{array}\right] \mid \xi \in \partial^{c} \varphi(\bar{x})\right\} \cup\left\{\nabla g_{i}(\bar{x}, \bar{y}) \mid i \in \bar{I}^{g}\right\}
$$

reduces to zero. Then, $(\bar{x}, \bar{y})$ is a strict local minimizer of (BPP).

Proof. The properties of Clarke's subdifferential can be used in order to see that

$$
\begin{aligned}
Q=\{\nabla F(\bar{x}, \bar{y})\} & \cup\left\{\nabla G_{i}(\bar{x}, \bar{y}) \mid i \in \bar{I}^{G}\right\} \\
& \cup\left\{\nabla f(\bar{x}, \bar{y})+\left[\begin{array}{l}
\tilde{\xi} \\
0
\end{array}\right] \mid \tilde{\xi} \in \partial^{c}(-\varphi)(\bar{x})\right\} \cup\left\{\nabla g_{i}(\bar{x}, \bar{y}) \mid i \in \bar{I}^{g}\right\}
\end{aligned}
$$

holds true. Since $Q^{\circ}=\{0\}$ is valid by assumption, the system (4.1a), (4.1b), (4.1d), and

$$
\nabla f(\bar{x}, \bar{y})^{\top} d+\max \left\{\tilde{\xi}^{\top} \delta_{x} \mid \tilde{\xi} \in \partial^{c}(-\varphi)(\bar{x})\right\} \leq 0
$$

does not possess a nontrivial solution $d:=\left(\delta_{x}, \delta_{y}\right) \in \mathbb{R}^{n} \times \mathbb{R}^{m}$. Observing that $-\varphi$ is Clarke-regular and, therefore, directionally differentiable, at $\bar{x}$, we particularly have

$$
\max \left\{\tilde{\xi}^{\top} \delta_{x} \mid \tilde{\xi} \in \partial^{c}(-\varphi)(\bar{x})\right\}=(-\varphi)^{\circ}\left(\bar{x} ; \delta_{x}\right)=(-\varphi)^{\prime}\left(\bar{x} ; \delta_{x}\right)=-\varphi^{\prime}\left(\bar{x} ; \delta_{x}\right)=-\varphi^{+}\left(\bar{x} ; \delta_{x}\right) .
$$

Thus, (4.1) does not possess a nontrivial solution. Due to Theorem 4.2, the assertion follows.

Clearly, the assumptions on the function $\varphi$ which are postulated in Theorem 4.7 trivially hold whenever $\varphi$ is locally finite and concave around the reference point. Exemplary, assume for a moment that the lower level problem is given by the simple parametric linear program

$$
\min _{y}\left\{(A x+c)^{\top} y \mid B y \leq b\right\}
$$

for matrices $A \in \mathbb{R}^{m \times n}, B \in \mathbb{R}^{q \times m}, b \in \mathbb{R}^{q}$, and $c \in \mathbb{R}^{m}$. Then, the associated optimal value function is concave and one obtains

$$
\partial^{c} \varphi(\bar{x})=A^{\top} S(\bar{x})=\left\{A^{\top} y \mid B y \leq b, B^{\top} p+A \bar{x}+c=0, p \geq 0, p^{\top}(B y-b)=0\right\}
$$

by strong duality of linear programming whenever $\bar{x} \in \operatorname{int} \operatorname{dom} S$ holds true, see Section 5.2 as well. This means that $Q$ in (4.2) is easily given in terms of initial problem data in this case.

We would like to point the reader's attention to the observation that the polar cone of the set $Q$ given in (4.2) reduces to $\{0\}$ whenever $0 \in \operatorname{int} \operatorname{conv} Q$ holds which might be easier to check than calculating the overall cone $Q^{\circ}$ or solving the system (4.1). 
Example 4.8. We revisit Example 4.3 w.r.t. the point $(\bar{x}, \bar{y}):=(0,0)$ in the light of Theorem 4.7. This is possible since $\varphi$ is a concave function. The set $Q$ is given by

$$
Q=\left\{\left(\begin{array}{l}
3 \\
2
\end{array}\right)\right\} \cup\left\{\left(\begin{array}{l}
\tilde{\xi} \\
0
\end{array}\right) \mid-1 \leq \tilde{\xi} \leq 0\right\} \cup\left\{\left(\begin{array}{c}
0 \\
-1
\end{array}\right)\right\}
$$

in this context. Given that $0 \in \operatorname{int} \operatorname{conv} Q$ holds, $Q^{\circ}$ reduces to the origin.

\subsection{STABLE UNIQUE LOWER LEVEL SOLUTIONS}

Let $M_{u}:=\left\{(x, y) \in \mathbb{R}^{n} \times \mathbb{R}^{m} \mid G(x, y) \leq 0\right\}$ denote the upper level feasible set. Clearly, we have $M=\operatorname{gph} S \cap M_{u}$ which yields

$$
\mathcal{T}_{M}(x, y) \subseteq \mathcal{T}_{\text {gph } S}(x, y) \cap \mathcal{T}_{M_{u}}(x, y)
$$

for each $(x, y) \in M$, see [2, Table 4.1]. Clearly, $\mathcal{T}_{M_{u}}(x, y)$ can be expressed via the associated linearized tangent cone as long as at least ACQ is valid at $(x, y)$ w.r.t. $M_{u}$. Thus, it remains to approximate $\mathcal{T}_{\text {gph } S}(x, y)$ from above.

In this section, we consider the situation where $S$ is locally single-valued, Lipschitz continuous, and directionally differentiable around a reference point $\bar{x}$, i.e. there is a neighborhood $U \subseteq \mathbb{R}^{n}$ of $\bar{x}$ and a locally Lipschitz continuous as well as directionally differentiable function $s: U \rightarrow \mathbb{R}^{m}$ satisfying $S(x)=\{s(x)\}$ for all $x \in U$. This can be guaranteed if the lower level problem is convex w.r.t. $y$ and satisfies LMFCQ, LCRCQ, as well as a strong second-order sufficient optimality condition at $(\bar{x}, \bar{y}) \in \operatorname{gph} S$, and under these assumptions, the directional derivative of $s$ can be computed as the solution of a quadratic optimization problem which is given in terms of initial problem data, see [35, Theorem 2] for details. Anyway, exploiting the directional differentiability of $s$ at $\bar{x}$, we have

$$
\mathcal{T}_{\text {gph } S}(\bar{x}, \bar{y})=\mathcal{T}_{\text {gph } s}(\bar{x}, s(\bar{x}))=\left\{\left(\delta_{x}, s^{\prime}\left(\bar{x} ; \delta_{x}\right)\right) \in \mathbb{R}^{n} \times \mathbb{R}^{m} \mid \delta_{x} \in \mathbb{R}^{n}\right\}
$$

by standard arguments. This leads to the following sufficient optimality condition for (BPP) which recovers [11, Theorem 4.2] and the considerations in [16, Section 2].

Theorem 4.9. Fix a feasible point $(\bar{x}, \bar{y}) \in \mathbb{R}^{n} \times \mathbb{R}^{m}$ of (BPP). Assume that there is a neighborhood $U \subseteq \mathbb{R}^{n}$ of $\bar{x}$ and a locally Lipschitz continuous, directionally differentiable function $s: U \rightarrow \mathbb{R}^{m}$ such that $S(x)=\{s(x)\}$ is valid for all $x \in U$. Furthermore, assume that the system

$$
\begin{aligned}
\nabla_{x} F(\bar{x}, \bar{y})^{\top} \delta_{x}+\nabla_{y} F(\bar{x}, \bar{y})^{\top} s^{\prime}\left(\bar{x} ; \delta_{x}\right) & \leq 0, \\
\nabla_{x} G_{i}(\bar{x}, \bar{y})^{\top} \delta_{x}+\nabla_{y} G_{i}(\bar{x}, \bar{y})^{\top} s^{\prime}\left(\bar{x} ; \delta_{x}\right) & \leq 0 \quad \forall i \in \bar{I}^{G}
\end{aligned}
$$

does not possess a nontrivial solution $\delta_{x} \in \mathbb{R}^{n}$. Then, $(\bar{x}, \bar{y})$ is a strict local minimizer of (BPP).

Proof. Noting that $\mathcal{T}_{M}(\bar{x}, \bar{y})$ can be approximated from above by the set $\mathcal{T}_{\text {gph } S}(\bar{x}, \bar{y}) \cap \mathcal{T}_{M_{u}}(\bar{x}, \bar{y})$ which admits the representation

$$
\left\{\left(\delta_{x}, s^{\prime}\left(\bar{x} ; \delta_{x}\right)\right) \in \mathbb{R}^{n} \times \mathbb{R}^{m} \mid \nabla_{x} G_{i}(\bar{x}, \bar{y})^{\top} \delta_{x}+\nabla_{y} G_{i}(\bar{x}, \bar{y})^{\top} s^{\prime}\left(\bar{x} ; \delta_{x}\right) \leq 0 \forall i \in \bar{I}^{G}\right\}
$$

due to the above considerations, this is an immediate consequence of Lemma 4.1.

\subsection{VARIATIONAL ANALYSIS APPROACH}

In this section, we want to exploit the decomposition (4.3) without assuming that $S$ is locally singlevalued around the reference point. Therefore, let us impose the following assumption for the remaining part of this section. 
Assumption 4.10. For each $x \in \mathbb{R}^{n}, f(x, \cdot): \mathbb{R}^{m} \rightarrow \mathbb{R}$ is convex. Furthermore, the function $g$ is independent of $x$ and componentwise convex. Thus, it is reasonable to set $K:=\left\{y \in \mathbb{R}^{m} \mid g(y) \leq 0\right\}$.

The above assumption allows us to interpret $S$ as the solution map associated with an equilibrium problem in the following sense:

$$
\forall x \in \mathbb{R}^{n}: \quad S(x)=\left\{y \in \mathbb{R}^{m} \mid-\nabla_{y} f(x, y) \in \widehat{\mathcal{N}}_{K}(y)\right\} .
$$

This leads to

$$
\operatorname{gph} S=\left\{(x, y) \in \mathbb{R}^{n} \times \mathbb{R}^{m} \mid\left(y,-\nabla_{y} f(x, y)\right) \in \operatorname{gph} \widehat{\mathcal{N}}_{K}\right\}
$$

where $\widehat{\mathcal{N}}_{K}: \mathbb{R}^{m} \rightrightarrows \mathbb{R}^{m}$ represents the (regular) normal cone mapping associated with the set $K$, which assigns to each $y \in \mathbb{R}^{m}$ the set $\widehat{\mathcal{N}}_{K}(y)$. By convexity and closedness of $K$, gph $\widehat{\mathcal{N}}_{K}$ is closed as well. Exploiting the preimage rule from [38, Theorem 6.31] as well as the fact that metric subregularity of feasibility maps is sufficient for some generalized ACQ to hold, see [26, Proposition 5], we obtain the following result.

Lemma 4.11. For $(\bar{x}, \bar{y}) \in \operatorname{gph} S$, we have

$$
\mathcal{T}_{\mathrm{gph} S}(\bar{x}, \bar{y}) \subseteq\left\{\left(\delta_{x}, \delta_{y}\right) \in \mathbb{R}^{n} \times \mathbb{R}^{m} \mid\left(\delta_{y},-\nabla_{y x}^{2} f(\bar{x}, \bar{y}) \delta_{x}-\nabla_{y y}^{2} f(\bar{x}, \bar{y}) \delta_{y}\right) \in \mathcal{T}_{\operatorname{gph} \widehat{N}_{K}}\left(\bar{y},-\nabla_{y} f(\bar{x}, \bar{y})\right)\right\} .
$$

Equality holds whenever the feasibility mapping

$$
\mathbb{R}^{n} \times \mathbb{R}^{m} \ni(x, y) \mapsto \operatorname{gph} \widehat{\mathcal{N}}_{K}-\left\{\left(y,-\nabla_{y} f(x, y)\right)\right\} \subseteq \mathbb{R}^{m} \times \mathbb{R}^{m}
$$

is metrically subregular at $(\bar{x}, \bar{y}, 0,0)$.

Recently, some progress has been made regarding the characterization of the tangent cone to the graph of $\widehat{\mathcal{N}}_{K}$, see [24, Section 4]. Thus, combining (4.3) with Lemma 4.11, Theorem 4.2 allows the derivation of a first-order sufficient optimality condition for (BPP).

Theorem 4.12. Let $(\bar{x}, \bar{y}) \in \mathbb{R}^{n} \times \mathbb{R}^{m}$ be a feasible point of (BPP). Assume for each $u \in \mathcal{L}_{K}(\bar{y}) \backslash\{0\}$ that

$$
0=\nabla g(\bar{y})^{\top} \lambda, \lambda \geq 0, \lambda^{\top} g(\bar{y})=0, \sum_{i=1}^{q} \lambda_{i} u^{\top} \nabla^{2} g_{i}(\bar{y}) u \geq 0 \quad \Longrightarrow \quad \lambda=0 .
$$

Finally, suppose that the system (4.1a), (4.1b), and

$$
\begin{aligned}
\nabla_{y x}^{2} f(\bar{x}, \bar{y}) \delta_{x}+\nabla_{y y}^{2} f(\bar{x}, \bar{y}) \delta_{y}+\kappa \nabla_{y} f(\bar{x}, \bar{y})+\sum_{i=1}^{q}\left(\lambda_{i} \nabla^{2} g_{i}(\bar{y}) \delta_{y}+\mu_{i} \nabla g_{i}(\bar{y})\right) & =0, \\
\nabla_{y} f(\bar{x}, \bar{y})+\nabla g(\bar{y})^{\top} \lambda=0, \quad \lambda \geq 0, \quad \lambda^{\top} g(\bar{y}) & =0, \\
\mu \geq 0, \quad \mu^{\top} g(\bar{y})=0, \quad \mu^{\top} \nabla g(\bar{y}) \delta_{y} & =0, \\
\nabla g_{i}(\bar{y})^{\top} \delta_{y} & \leq 0 \quad i \in \bar{I}^{g}, \\
\nabla f_{y}(\bar{x}, \bar{y})^{\top} \delta_{y} & =0
\end{aligned}
$$

does not possess a solution $\left(\delta_{x}, \delta_{y}, \lambda, \kappa, \mu\right) \in \mathbb{R}^{n} \times \mathbb{R}^{m} \times \mathbb{R}^{q} \times \mathbb{R} \times \mathbb{R}^{q}$ which satisfies $\left(\delta_{x}, \delta_{y}\right) \neq(0,0)$. Then, $(\bar{x}, \bar{y})$ is a strict local minimizer of (BPP).

Proof. Due to [24, Proposition 3, Theorem 1], the postulated condition (4.6) guarantees that we have

$$
\mathcal{T}_{\text {gph } \widehat{\mathcal{N}}_{K}}\left(\bar{y},-\nabla_{y} f(\bar{x}, \bar{y})\right)=\left\{\left(r_{1}, r_{2}\right) \in \mathbb{R}^{m} \times \mathbb{R}^{m} \mid \exists \lambda \in \Lambda(\bar{x}, \bar{y}): r_{2}-\sum_{i=1}^{q} \lambda_{i} \nabla^{2} g_{i}(\bar{y}) r_{1} \in \widehat{\mathcal{N}}_{C^{L}(\bar{x}, \bar{y})}\left(r_{1}\right)\right\}
$$


where $\Lambda(\bar{x}, \bar{y})$ denotes the set of lower level Lagrange multipliers and $C^{L}(\bar{x}, \bar{y})$ represents the lower level critical cone, see (3.2). Noting that $C^{L}(\bar{x}, \bar{y})$ is a closed, convex, polyhedral cone, we have

$$
\begin{aligned}
\widehat{\mathcal{N}}_{C^{L}(\bar{x}, \bar{y})}\left(\delta_{y}\right) & =C^{L}(\bar{x}, \bar{y})^{\circ} \cap\left\{\delta_{y}\right\}^{\perp} \\
& =\left\{\kappa \nabla_{y} f(\bar{x}, \bar{y})+\sum_{i=1}^{q} \mu_{i} \nabla g_{i}(\bar{y}) \mid \kappa \in \mathbb{R}, \mu \geq 0, \mu^{\top} g(\bar{y})=0\right\} \cap\left\{\delta_{y}\right\}^{\perp} \\
& =\left\{\kappa \nabla_{y} f(\bar{x}, \bar{y})+\sum_{i=1}^{q} \mu_{i} \nabla g_{i}(\bar{y}) \mid \kappa \in \mathbb{R}, \mu \geq 0, \mu^{\top} g(\bar{y})=0, \mu^{\top} \nabla g(\bar{y}) \delta_{y}=0\right\}
\end{aligned}
$$

for each $\delta_{y} \in C^{L}(\bar{x}, \bar{y})$ by definition of the latter cone.

Clearly, for each $\left(\delta_{x}, \delta_{y}\right) \in \mathcal{T}_{M}(\bar{x}, \bar{y})$, we have (4.1b) and $\left(\delta_{x}, \delta_{y}\right) \in \mathcal{T}_{\text {gph } S}(\bar{x}, \bar{y})$ due to $(4.3)$ and Lemma 2.8. Combining Lemma 4.11 and the above considerations, $\left(\delta_{x}, \delta_{y}\right) \in \mathcal{T}_{\text {gph } S}(\bar{x}, \bar{y})$ implies that there are $\lambda, \mu \in \mathbb{R}^{q}$ and $\kappa \in \mathbb{R}$ satisfying (4.7). Thus, observing that each solution $\left(\delta_{x}, \delta_{y}, \lambda, \kappa, \mu\right)$ of (4.1a), (4.1b), and (4.7) satisfies $\left(\delta_{x}, \delta_{y}\right)=(0,0)$, there does not exist $\left(\delta_{x}, \delta_{y}\right) \in \mathcal{T}_{M}(\bar{x}, \bar{y})$ such that

$$
\nabla_{x} F(\bar{x}, \bar{y})^{\top} \delta_{x}+\nabla_{y} F(\bar{x}, \bar{y})^{\top} \delta_{y} \leq 0 .
$$

Hence, due to Lemma $4 \cdot 1,(\bar{x}, \bar{y})$ is a strict local minimizer of (BPP).

In the literature, (4.6) is referred to as Second-Order Sufficient Condition for Metric Subregularity, see, e.g., [22, Corollary 1]. Clearly, this condition is weaker than LMFCQ. Recall that the exploited estimate for $\mathcal{T}_{\text {gph } S}(\bar{x}, \bar{y})$ is sharp when the set-valued mapping in (4.5) is metrically subregular, see Lemma 4.11. In this case, the conditions in Theorem 4.12 are indeed of reasonable strength. Note that whenever $K$ is polyhedral, then gph $\widehat{\mathcal{N}}_{K}$ is the union of finitely many polyhedral sets. If, additionally, $\nabla_{y} f$ is affine jointly in $x$ and $y$, then the multifunction in (4.5) is a so-called polyhedral set-valued mapping, and this property ensures that it is metrically subregular everywhere on its graph, see [36, Proposition 1]. This setting is inherent for lower level problems of obstacle-type given by

$$
\min _{y}\left\{\frac{1}{2} y^{\top} A y-(B x+c)^{\top} y \mid \psi_{\ell} \leq y \leq \psi_{u}\right\}
$$

where $A \in \mathbb{R}^{m \times m}$ is symmetric and positive semidefinite, $B \in \mathbb{R}^{m \times n}$ as well as $c \in \mathbb{R}^{m}$ are chosen arbitrarily, and the lower and upper obstacle $\psi_{\ell}, \psi_{u} \in \mathbb{R}^{m}$ satisfy $\psi_{\ell}<\psi_{u}$, see [27, Section 4] as well.

Exploiting recent theory from [23], the results of Theorem 4.12 can be generalized to the setting where $K$ depends on $x$. In this case, some more restrictive assumptions have to be postulated and the system (4.7) gets far more complex.

The upcoming example depicts that, in contrast to Theorem 4.9, Theorem 4.12 is indeed capable of identifying local minimizers of (BPP) in situations where $S$ is not single-valued.

Example 4.13. Consider the bilevel optimization problem

$$
\min _{x, y}\{-x-y \mid-x+y \leq 1, y \in S(x)\}
$$

where $S: \mathbb{R} \rightrightarrows \mathbb{R}$ is the solution map of $\min _{y}\left\{x y^{2} \mid y \in[-1,1]\right\}$. Here, we have

$$
\forall x \in \mathbb{R}: \quad S(x)= \begin{cases}\{-1,1\} & x<0, \\ {[-1,1]} & x=0, \\ \{0\} & x>0 .\end{cases}
$$

We consider $(\bar{x}, \bar{y}):=(0,1)$. The associated system (4.1a), (4.1b), (4.7) reduces to

$$
-\delta_{x}-\delta_{y} \leq 0, \quad-\delta_{x}+\delta_{y} \leq 0, \quad 2 \delta_{x}+\mu=0, \quad \mu \geq 0, \quad \mu \delta_{y}=0, \quad \delta_{y} \leq 0,
$$

and one can easily check that all of its solutions satisfy $\delta_{x}=\delta_{y}=0$ which means that $(\bar{x}, \bar{y})$ is a strict local minimizer of the considered bilevel optimization problem, see Theorem 4.12. 


\subsection{KKT-APPROACH}

In this section, we want to exploit the lower level KKT-conditions for a detailed expression of the set $M$. This is a reasonable approach as long as the lower level problem is convex w.r.t. $y$ and sufficiently regular. Below, we specify these assumptions which have to hold throughout this section.

Assumption 4.14. For each $x \in \mathbb{R}^{n}, f(x, \cdot): \mathbb{R}^{m} \rightarrow \mathbb{R}$ is convex while $g(x, \cdot): \mathbb{R}^{m} \rightarrow \mathbb{R}^{q}$ is componentwise convex. Furthermore, at each point of gph $K$, a lower level constraint qualification holds.

Due to the postulated convexity and regularity assumptions, writing $(x, y) \in \operatorname{gph} S$ is equivalent to $(x, y) \in \operatorname{gph} K$ and $\Lambda(x, y) \neq \varnothing$. For subsequent use, we introduce the classical index sets

$$
\begin{aligned}
\bar{I}^{0-} & =I^{0-}(\bar{x}, \bar{y}, \bar{\lambda}):=\left\{i \in\{1, \ldots, q\} \mid \bar{\lambda}_{i}=0 \wedge g_{i}(\bar{x}, \bar{y})<0\right\}, \\
\bar{I}^{+0} & =I^{+0}(\bar{x}, \bar{y}, \bar{\lambda}):=\left\{i \in\{1, \ldots, q\} \mid \bar{\lambda}_{i}>0 \wedge g_{i}(\bar{x}, \bar{y})=0\right\}, \\
\bar{I}^{00} & =I^{00}(\bar{x}, \bar{y}, \bar{\lambda}):=\left\{i \in\{1, \ldots, q\} \mid \bar{\lambda}_{i}=0 \wedge g_{i}(\bar{x}, \bar{y})=0\right\},
\end{aligned}
$$

where $(\bar{x}, \bar{y}) \in \operatorname{gph} S$ and $\bar{\lambda} \in \Lambda(\bar{x}, \bar{y})$ are arbitrarily chosen. Clearly, $\bar{I}^{+0} \cup \bar{I}^{00}=\bar{I}^{g}$ holds by definition.

Due to the results from [13], we want to abstain from using the KKT-reformulation of the bilevel programming problem (BPP) since the surrogate mathematical program with complementarity constraints (MPCC for short) given by

$$
\min _{x, y, \lambda}\left\{F(x, y) \mid G(x, y) \leq 0, \nabla_{y} L(x, y, \lambda)=0,0 \leq \lambda \perp g(x, y) \leq 0\right\},
$$

where the lower level Lagrange multiplier is treated as an explicit variable, may possess artificial local minimizers which are not related to local minimizers of (BPP). Additionally, one has to check local optimality w.r.t. all associated lower level Lagrange multipliers for (KKT) in order to verify local optimality of the underlying feasible point of (BPP). For later use, however, let $M_{\mathrm{KKT}} \subseteq \mathbb{R}^{n} \times \mathbb{R}^{m} \times \mathbb{R}^{q}$ be the feasible set of (KKT). Then, we clearly have the relation $M=\Pi M_{\mathrm{KKT}}$, where $\Pi \in \mathbb{R}^{(n+m) \times(n+m+q)}$ is the projection matrix

$$
\Pi:=\left[\begin{array}{ccc}
I_{n} & \mathbb{O} & \mathbb{O} \\
\mathbb{O} & I_{m} & \mathbb{O}
\end{array}\right] .
$$

Now, Lemma 2.1 opens a way to compute an upper approximate of the tangent cone to $M$.

Lemma 4.15. Let $(\bar{x}, \bar{y}) \in \mathbb{R}^{n} \times \mathbb{R}^{m}$ be a feasible point of (BPP) and let $\bar{\lambda} \in \Lambda(\bar{x}, \bar{y})$ be chosen such that the condition

$$
\left.\begin{array}{l}
0=\nabla_{y} g(\bar{x}, \bar{y})^{\top} \mu, \\
\forall i \notin \bar{I}^{g}: \mu_{i}=0, \\
\forall i \in \bar{I}^{00}: \mu_{i} \geq 0
\end{array}\right\} \Longrightarrow \mu=0
$$

holds true. Then, we have

$$
\mathcal{T}_{M}(\bar{x}, \bar{y}) \subseteq \Pi\left\{\left(\delta_{x}, \delta_{y}, \delta_{\lambda}\right) \mid \begin{array}{rl}
\nabla_{x} G_{i}(\bar{x}, \bar{y})^{\top} \delta_{x}+\nabla_{y} G_{i}(\bar{x}, \bar{y})^{\top} \delta_{y} \leq 0 & i \in \bar{I}^{G} \\
\nabla_{y x}^{2} L(\bar{x}, \bar{y}, \bar{\lambda}) \delta_{x}+\nabla_{y y}^{2} L(\bar{x}, \bar{y}, \bar{\lambda}) \delta_{y}+\nabla_{y} g(\bar{x}, \bar{y})^{\top} \delta_{\lambda}=0 & \\
\left(\delta_{\lambda}\right)_{i}=0 & i \in \bar{I}^{0-} \\
\nabla_{x} g_{i}(\bar{x}, \bar{y})^{\top} \delta_{x}+\nabla_{y} g_{i}(\bar{x}, \bar{y})^{\top} \delta_{y}=0 & i \in \bar{I}^{+0} \\
0 \leq\left(\delta_{\lambda}\right)_{i} \perp \nabla_{x} g_{i}(\bar{x}, \bar{y})^{\top} \delta_{x}+\nabla_{y} g_{i}(\bar{x}, \bar{y})^{\top} \delta_{y} \leq 0 \quad i \in \bar{I}^{00}
\end{array}\right\} .
$$

Proof. Due to Lemma 2.1, we have

$$
\mathcal{T}_{M}(\bar{x}, \bar{y})=\mathcal{T}_{\Pi M_{\mathrm{KKT}}}(\bar{x}, \bar{y})=\operatorname{cl}\left(\Pi \mathcal{T}_{M_{\mathrm{KKT}}}(\bar{x}, \bar{y}, \bar{\lambda})\right)
$$


provided that the constraint qualification

$$
\operatorname{ker} \Pi \cap \mathcal{T}_{M_{\mathrm{KKT}}}(\bar{x}, \bar{y}, \bar{\lambda})=\{0\}
$$

holds true. Obviously, $\operatorname{ker} \Pi=\{0\} \times\{0\} \times \mathbb{R}^{q}$ is valid. It is well known from [19, Lemma 3.2] that the so-called MPCC-tailored linearization cone

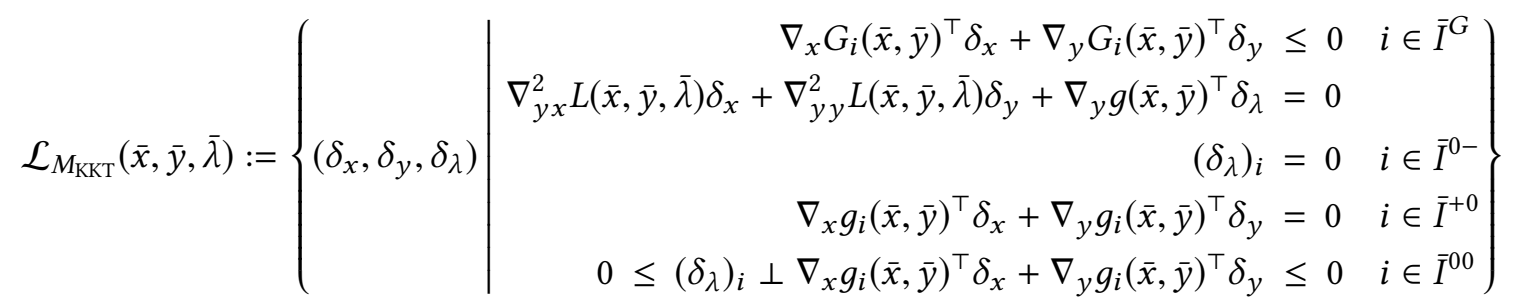

always provides an upper estimate of $\mathcal{T}_{M_{\mathrm{KKT}}}(\bar{x}, \bar{y}, \bar{\lambda})$.

Let us note that any vector $\left(\delta_{x}, \delta_{y}, \delta_{\lambda}\right) \in \operatorname{ker} \Pi \cap \mathcal{L}_{M_{\mathrm{KKT}}}(\bar{x}, \bar{y}, \bar{\lambda})$, by definition, satisfies $\delta_{x}=\delta_{y}=0$, $\nabla_{y} g(\bar{x}, \bar{y})^{\top} \delta_{\lambda}=0,\left(\delta_{\lambda}\right)_{i}=0$ for all $i \in \bar{I}^{0-}=\{1, \ldots, q\} \backslash \bar{I}^{g}$, and $\left(\delta_{\lambda}\right)_{i} \geq 0$ for all $i \in \bar{I}^{00}$. Now, the validity of (4.9) guarantees $\delta_{\lambda}=0$. Particularly, (4.10) holds as well. As a consequence, we have

$$
\mathcal{T}_{M}(\bar{x}, \bar{y})=\operatorname{cl}\left(\Pi \mathcal{T}_{M_{\mathrm{KKT}}}(\bar{x}, \bar{y}, \bar{\lambda})\right) \subseteq \operatorname{cl}\left(\Pi \mathcal{L}_{M_{\mathrm{KKT}}}(\bar{x}, \bar{y}, \bar{\lambda})\right)=\Pi \mathcal{L}_{M_{\mathrm{KKT}}}(\bar{x}, \bar{y}, \bar{\lambda})
$$

since $\mathcal{L}_{M_{\mathrm{KKT}}}(\bar{x}, \bar{y}, \bar{\lambda})$ is the union of finitely many polyhedral cones which implies that $\Pi \mathcal{L}_{M_{\mathrm{KKT}}}(\bar{x}, \bar{y}, \bar{\lambda})$ possesses the same property and, thus, is closed. This shows the desired estimate.

Let us comment on the above lemma in the subsequent remarks.

Remark 4.16. In the literature, see [29, 46], condition (4.9) is referred to as Strict Mangasarian-Fromovitz Condition. It is well known that this condition, which cannot be called a constraint qualification since it depends explicitly on a Lagrange multiplier $\bar{\lambda}$ and, thus, implicitly on the lower level objective functional, already implies the uniqueness of the multiplier, i.e. $\Lambda(\bar{x}, \bar{y})$ reduces to the singleton $\{\bar{\lambda}\}$. It can be easily checked that validity of (4.9) is inherent whenever LLICQ holds at the reference point, but (4.9) may hold in situations where LLICQ is violated, see [29, Example 2.2].

Remark 4.17. Following the lines of the proof of Lemma 4.15, the provided estimate for $\mathcal{T}_{M}(\bar{x}, \bar{y})$ is exact whenever the relation $\mathcal{T}_{M_{\mathrm{KKT}}}(\bar{x}, \bar{y}, \bar{\lambda})=\mathcal{L}_{M_{\mathrm{KKT}}}(\bar{x}, \bar{y}, \bar{\lambda})$ is valid. In the literature on MPCCs, this condition is refereed to as MPCC-ACQ, see, e.g., [19, Definition 3.1], and is a comparatively weak MPCC-tailored constraint qualification. It holds, e.g., when $G$ is affine and the lower level problem is of obstacle-type (4.8), see [19, Theorem 3.2]. In this setting, LLICQ is valid at all lower level feasible points and, thus, (4.9) is valid as well, i.e. Lemma 4.15 provides a precise formula for the tangent cone to $M$ at $(\bar{x}, \bar{y})$.

Combining Lemmas 4.1 and 4.15, we have the following first-order sufficient optimality condition.

Theorem 4.18. Let $(\bar{x}, \bar{y}) \in \mathbb{R}^{n} \times \mathbb{R}^{m}$ be a feasible point of (BPP). Furthermore, fix $\bar{\lambda} \in \Lambda(\bar{x}, \bar{y})$ satisfying (4.9). Finally, suppose that the system (4.1a), (4.1b),

$$
\begin{array}{rl}
\nabla_{y x}^{2} L(\bar{x}, \bar{y}, \bar{\lambda}) \delta_{x}+\nabla_{y y}^{2} L(\bar{x}, \bar{y}, \bar{\lambda}) \delta_{y}+\nabla_{y} g(\bar{x}, \bar{y})^{\top} \delta_{\lambda}=0, & \\
\left(\delta_{\lambda}\right)_{i}=0 & i \in \bar{I}^{0-}, \\
\nabla_{x} g_{i}(\bar{x}, \bar{y})^{\top} \delta_{x}+\nabla_{y} g_{i}(\bar{x}, \bar{y})^{\top} \delta_{y}=0 & i \in \bar{I}^{+0}, \\
0 \leq\left(\delta_{\lambda}\right)_{i} \perp \nabla_{x} g_{i}(\bar{x}, \bar{y})^{\top} \delta_{x}+\nabla_{y} g_{i}(\bar{x}, \bar{y})^{\top} \delta_{y} \leq 0 & i \in \bar{I}^{00}
\end{array}
$$

does not possess a nontrivial solution $\left(\delta_{x}, \delta_{y}, \delta_{\lambda}\right)$. Then, $(\bar{x}, \bar{y})$ is a strict local minimizer of (BPP).

In the subsequent remarks, we comment on this theorem and its assumptions. 
Remark 4.19. Under validity of (4.9), each solution $\left(\delta_{x}, \delta_{y}, \delta_{\lambda}\right)$ of the system (4.1a), (4.1b), (4.11) which satisfies $\left(\delta_{x}, \delta_{y}\right)=(0,0)$ already satisfies $\delta_{\lambda}=0$. Thus, one could equivalently state the assertion of Theorem 4.18 via postulating that the system (4.1a), (4.1b), (4.11) does not possess a solution $\left(\delta_{x}, \delta_{y}, \delta_{\lambda}\right)$ which satisfies $\left(\delta_{x}, \delta_{y}\right) \neq(0,0)$, and the latter seems to be natural in light of $\mathcal{T}_{M}(\bar{x}, \bar{y}) \subseteq \mathbb{R}^{n} \times \mathbb{R}^{m}$ and Lemma 4.1.

Remark 4.20. Exploiting [19, Lemma 3.2], one can show that the assumptions of Theorem 4.18 guarantee that $(\bar{x}, \bar{y}, \bar{\lambda})$ is a strict local minimizer of (KKT). Observing that the validity of (4.9) implies that $\Lambda(\bar{x}, \bar{y})=\{\bar{\lambda}\}$ holds, see Remark 4.16, we can use [13, Theorem 3.2] in order to infer that $(\bar{x}, \bar{y})$ is a strict local minimizer of (BPP). This provides another proof strategy for Theorem 4.18.

$\operatorname{Remark} 4$ 4.21. Let us note that for each local minimizer $(\tilde{x}, \tilde{y})$ of (BPP) such that $\Lambda(\tilde{x}, \tilde{y})$ is not a singleton, the point $(\tilde{x}, \tilde{y}, \tilde{\lambda})$ cannot be a strict local minimizer of (KKT) for each $\tilde{\lambda} \in \Lambda(\tilde{x}, \tilde{y})$ since the objective functional of (KKT) does not depend on $\lambda$. Thus, the KKT-approach is not suited for identifying strict local minimizer of (BPP) with nonunique lower level multiplier since first- (or second-) order sufficient optimality conditions imply local linear (or quadratic) growth of the objective functional around the point of interest. In this regard, the restriction to situations where the lower level Lagrange multiplier is uniquely determined seems to be quite natural in the context of this section. Noting that the Strict Mangasarian-Fromovitz Condition (4.9) is the weakest condition which guarantees this, see [29, Proposition 1.1], the assumptions of Theorem 4.18 are quite reasonable.

Example 4.22. We consider the bilevel programming problem from Example 4.13 at $(\bar{x}, \bar{y}):=(0,1)$. The associated lower level Lagrange multiplier is uniquely determined and given by $\bar{\lambda}=(0,0)$. This yields $\bar{I}^{0-}=\{1\}$ and $\bar{I}^{00}=\{2\}$ where we used $g_{1}(y):=-y-1$ and $g_{2}(y):=y-1$ for all $y \in \mathbb{R}$. The associated system (4.1a), (4.1b), (4.11) reads as

$$
-\delta_{x}-\delta_{y} \leq 0, \quad \delta_{x}-\delta_{y} \leq 0, \quad 2 \delta_{x}-\left(\delta_{\lambda}\right)_{1}+\left(\delta_{\lambda}\right)_{2}=0, \quad\left(\delta_{\lambda}\right)_{1}=0, \quad 0 \leq\left(\delta_{\lambda}\right)_{2} \perp \delta_{y} \leq 0 .
$$

and, obviously, does not possess a nontrivial solution. Thus, Theorem 4.18 correctly identifies the strict local minimizer $(\bar{x}, \bar{y})$.

\section{A SECOND-ORDER SUFFICIENT OPTIMALITY CONDITION}

In this section, we are going to derive a second-order sufficient optimality condition for (BPP) which is based on (LLVF). Therefore, we will make use of the second-order directional derivative of the optimal value function $\varphi$. First, we are going to state a rather general result which is, afterwards, specified to different settings where the assumptions are partially inherent or can be easily verified.

Let $(\bar{x}, \bar{y}) \in \mathbb{R}^{n} \times \mathbb{R}^{m}$ be a feasible point of (BPP) and assume that $\varphi$ is directionally differentiable at $\bar{x}$. For subsequent use, we introduce the linearization cone

$$
\mathcal{L}_{M}^{\varphi}(\bar{x}, \bar{y}):=\left\{\begin{array}{l|l}
d:=\left(\delta_{x}, \delta_{y}\right) \in \mathbb{R}^{n} \times \mathbb{R}^{m} \mid \begin{array}{r}
\nabla G_{i}(\bar{x}, \bar{y})^{\top} d \leq 0 \\
\nabla f(\bar{x}, \bar{y})^{\top} d-\varphi^{\prime}\left(\bar{x} ; \delta_{x}\right) \leq 0 \\
\nabla g_{i}(\bar{x}, \bar{y})^{\top} d \leq 0 \quad i \in \bar{I}^{g}
\end{array}
\end{array}\right\}
$$

and the critical cone

$$
C_{M}^{\varphi}(\bar{x}, \bar{y}):=\mathcal{L}_{M}^{\varphi}(\bar{x}, \bar{y}) \cap\left\{d \in \mathbb{R}^{n} \times \mathbb{R}^{m} \mid \nabla F(\bar{x}, \bar{y})^{\top} d \leq 0\right\},
$$

of (LLVF) at $(\bar{x}, \bar{y})$. Note that $\mathcal{L}_{M}^{\varphi}(\bar{x}, \bar{y})$ and $C_{M}^{\varphi}(\bar{x}, \bar{y})$ do not need to be convex as soon as the function $\varphi$ is nonsmooth at the point $\bar{x}$.

Remark 5.1. Let $(\bar{x}, \bar{y}) \in \mathbb{R}^{n} \times \mathbb{R}^{m}$ be a feasible point of (BPP) and let $\varphi$ be locally Lipschitz continuous and directionally differentiable at $\bar{x}$. Furthermore, assume that at least ACQ holds for the set

$$
\left\{(x, y) \in \mathbb{R}^{n} \times \mathbb{R}^{m} \mid g(x, y) \leq 0\right\}
$$


at $(\bar{x}, \bar{y})$. Noting that $(\bar{x}, \bar{y})$ is a global minimizer of

$$
\min _{x, y}\{f(x, y)-\varphi(x) \mid g(x, y) \leq 0\}
$$

by definition of $\varphi$, Lemma 2.5 and the validity of ACQ yield

$$
\forall d:=\left(\delta_{x}, \delta_{y}\right) \in \mathbb{R}^{n} \times \mathbb{R}^{m}: \quad\left(\forall i \in \bar{I}^{g}: \nabla g_{i}(\bar{x}, \bar{y})^{\top} d \leq 0\right) \Longrightarrow \nabla f(\bar{x}, \bar{y})^{\top} d-\varphi^{\prime}\left(\bar{x} ; \delta_{x}\right) \geq 0 .
$$

Thus, in this situation, the linearization cone $\mathcal{L}_{M}^{\varphi}(\bar{x}, \bar{y})$ possesses the representation

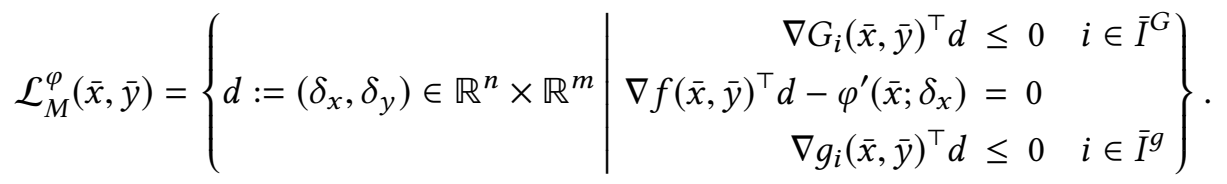

The upcoming example depicts that the statement of Remark 5.1 does not generally hold in the absence of a constraint qualification.

Example 5.2. Let us consider the lower level problem

$$
S(x):=\underset{y}{\operatorname{argmin}}\left\{y_{1} \mid y_{1}^{2}+y_{2} \leq 0, y_{1}^{2}+\left(y_{2}-1\right)^{2} \leq 1+x^{2}\right\} .
$$

One can check that it holds

$$
\forall x \in \mathbb{R}: \quad S(x)=\left\{\left(-\sqrt{\sqrt{2.25+x^{2}}-1.5}, 1.5-\sqrt{2.25+x^{2}}\right)\right\} .
$$

Thus, the optimal value function $\varphi: \mathbb{R} \rightarrow \mathbb{R}$ is given by

$$
\forall x \in \mathbb{R}: \quad \varphi(x)=-\sqrt{\sqrt{2.25+x^{2}}-1.5}=-\frac{|x|}{\sqrt{\sqrt{2.25+x^{2}}+1.5}} .
$$

Let us consider $(\bar{x}, \bar{y}):=(0,0) \in \operatorname{gph} S$. At $\bar{x}, \varphi$ is locally Lipschitz continuous as well as directionally differentiable, and it holds

$$
\forall \delta_{x} \in \mathbb{R}: \quad \varphi^{\prime}\left(\bar{x} ; \delta_{x}\right)=-\frac{1}{\sqrt{3}}\left|\delta_{x}\right| .
$$

The linearization cone to the set given in (5.2) at $(\bar{x}, \bar{y})$ is given by $\left\{\left(\delta_{x}, \delta_{y, 1}, \delta_{y, 2}\right) \in \mathbb{R}^{3} \mid \delta_{y, 2}=0\right\}$. Particularly, the direction $(0,-1,0)$ belongs to this cone although it is not a tangent direction, i.e. ACQ does not hold at $(\bar{x}, \bar{y})$ for the set in (5.2). Using this direction, one can check that (5.3) is clearly violated, and, consequently, the potential representation of the linearization cone from Remark 5.1 is strictly smaller than the original linearization cone from (5.1).

Now, we are in position to state the second-order sufficient condition of interest. Our arguments here are inspired by [39, Theorem 4.1].

Theorem 5.3. Let $(\bar{x}, \bar{y}) \in \mathbb{R}^{n} \times \mathbb{R}^{m}$ be feasible to (BPP) such that at least ACQ holds for the set in (5.2) at $(\bar{x}, \bar{y})$. Let $\varphi$ be locally Lipschitzian and second-order directionally differentiable at $\bar{x}$. Moreover, let $-\varphi$ be second-order epi-regular at $\bar{x}$. Finally, assume that we have

$$
\forall d \in \mathcal{L}_{M}^{\varphi}(\bar{x}, \bar{y}): \quad \nabla F(\bar{x}, \bar{y})^{\top} d \geq 0,
$$

and that the optimization problem

$$
\min _{w=\left(\omega_{x}, \omega_{y}\right), \alpha}\left\{\alpha \mid \begin{array}{rl}
\nabla F(\bar{x}, \bar{y})^{\top} w+d^{\top} \nabla^{2} F(\bar{x}, \bar{y}) d & \leq \alpha \\
\nabla G_{i}(\bar{x}, \bar{y})^{\top} w+d^{\top} \nabla^{2} G_{i}(\bar{x}, \bar{y}) d & \leq \alpha \quad i \in \bar{I}^{G}(d) \\
\nabla f(\bar{x}, \bar{y})^{\top} w+d^{\top} \nabla^{2} f(\bar{x}, \bar{y}) d-\varphi^{\prime \prime}\left(\bar{x} ; \delta_{x}, \omega_{x}\right) & \leq \alpha \\
\nabla g_{i}(\bar{x}, \bar{y})^{\top} w+d^{\top} \nabla^{2} g_{i}(\bar{x}, \bar{y}) d & \leq \alpha \quad i \in \bar{I}^{g}(d)
\end{array}\right\}
$$


possesses a positive optimal objective value for each $d \in C_{M}^{\varphi}(\bar{x}, \bar{y}) \backslash\{0\}$. Here, we use

$$
\bar{I}^{G}(d):=\left\{i \in \bar{I}^{G} \mid \nabla G_{i}(\bar{x}, \bar{y})^{\top} d=0\right\} \quad \text { and } \quad \bar{I}^{g}(d):=\left\{i \in \bar{I}^{g} \mid \nabla g_{i}(\bar{x}, \bar{y})^{\top} d=0\right\} .
$$

Then, $(\bar{x}, \bar{y})$ is a strict local minimizer of (BPP) which satisfies the second-order growth condition.

Proof. For the proof, we exploit the functional $\psi: \mathbb{R}^{n} \times \mathbb{R}^{m} \rightarrow \overline{\mathbb{R}}$ defined, for all $(x, y) \in \mathbb{R}^{n} \times \mathbb{R}^{m}$, by

$$
\psi(x, y):=\max \left\{F(x, y)-F(\bar{x}, \bar{y}), G_{1}(x, y), \ldots, G_{p}(x, y), f(x, y)-\varphi(x), g_{1}(x, y), \ldots, g_{q}(x, y)\right\} .
$$

As a first step of the proof, we show that whenever the second-order growth condition holds for $\psi$ at $(\bar{x}, \bar{y})$, then it also holds for the optimization problem (LLVF) at $(\bar{x}, \bar{y})$. Thus, we assume that there are constants $\varepsilon>0$ and $c>0$ such that

$$
\forall(x, y) \in \mathbb{B}_{\varepsilon}(\bar{x}, \bar{y}): \quad \psi(x, y) \geq c\left(\|x-\bar{x}\|_{2}^{2}+\|y-\bar{y}\|_{2}^{2}\right)
$$

holds. Now, suppose that the second-order growth condition does not hold for (LLVF) at $(\bar{x}, \bar{y})$. Then, we find a sequence $\left\{\left(x_{k}, y_{k}\right)\right\}_{k \in \mathbb{N}} \subseteq M$ converging to $(\bar{x}, \bar{y})$ which satisfies

$$
\forall k \in \mathbb{N}: \quad F\left(x_{k}, y_{k}\right)<F(\bar{x}, \bar{y})+\frac{1}{k}\left(\left\|x_{k}-\bar{x}\right\|_{2}^{2}+\left\|y_{k}-\bar{y}\right\|_{2}^{2}\right) .
$$

By definition of $\psi$, this yields

$$
\forall k \in \mathbb{N}: \quad \psi\left(x_{k}, y_{k}\right) \leq \max \left\{F\left(x_{k}, y_{k}\right)-F(\bar{x}, \bar{y}), 0\right\}<\frac{1}{k}\left(\left\|x_{k}-\bar{x}\right\|_{2}^{2}+\left\|y_{k}-\bar{y}\right\|_{2}^{2}\right) ;
$$

clearly contradicting (5.6). Thus, the second-order growth condition needs to hold for (LLVF) at $(\bar{x}, \bar{y})$.

In the remaining part of the proof, we verify that $\psi$ indeed satisfies the second-order growth condition at $(\bar{x}, \bar{y})$ which, due to the above arguments, would yield the claim. This can be done with the aid of Proposition 2.10. Due to the assumptions of the theorem, we note that $\psi$ is locally Lipschitz continuous and second-order directionally differentiable at $(\bar{x}, \bar{y})$, see Lemma 2.2. Since $-\varphi$ is assumed to be secondorder epi-regular at $\bar{x}$, we can exploit Lemma 2.4 in order to see that $\psi$ is second-order epi-regular at $(\bar{x}, \bar{y})$. Noting that we have

$$
\begin{aligned}
& \psi^{\prime}((\bar{x}, \bar{y}) ; d)=\max \left\{\nabla F(\bar{x}, \bar{y})^{\top} d, \max \right.\left\{\nabla G_{i}(\bar{x}, \bar{y})^{\top} d \mid i \in \bar{I}^{G}\right\}, \\
&\left.\nabla f(\bar{x}, \bar{y})^{\top} d-\varphi^{\prime}\left(\bar{x} ; \delta_{x}\right), \max \left\{\nabla g_{i}(\bar{x}, \bar{y})^{\top} d \mid i \in \bar{I}^{g}\right\}\right\}
\end{aligned}
$$

for all $d:=\left(\delta_{x}, \delta_{y}\right) \in \mathbb{R}^{n} \times \mathbb{R}^{m}$ from Lemma 2.2 and the equality $f(\bar{x}, \bar{y})-\varphi(\bar{x})=0$ which holds by definition of $\varphi$, condition (5.4) implies $\psi^{\prime}((\bar{x}, \bar{y}) ; d) \geq 0$ for all $d \in \mathbb{R}^{n} \times \mathbb{R}^{m}$. Next, we observe that due to $(5.4)$, it holds

$$
C_{M}^{\varphi}(\bar{x}, \bar{y})=\left\{d \in \mathbb{R}^{n} \times \mathbb{R}^{m} \mid \psi^{\prime}((\bar{x}, \bar{y}) ; d)=0\right\} .
$$

Pick $d:=\left(\delta_{x}, \delta_{y}\right) \in C_{M}^{\varphi}(\bar{x}, \bar{y})$ arbitrarily. Due to (5.4), it holds $\nabla F(\bar{x}, \bar{y})^{\top} d=0$. Furthermore, Remark 5.1 guarantees $\nabla f(\bar{x}, \bar{y})^{\top} d-\varphi^{\prime}\left(\bar{x} ; \delta_{x}\right)=0$. Exploiting Lemma 2.2 again, we obtain

$$
\begin{aligned}
\psi^{\prime \prime}((\bar{x}, \bar{y}) ; d, w)=\max \{ & \nabla F(\bar{x}, \bar{y})^{\top} w+d^{\top} \nabla^{2} F(\bar{x}, \bar{y}) d, \\
& \max \left\{\nabla G_{i}(\bar{x}, \bar{y})^{\top} w+d^{\top} \nabla^{2} G_{i}(\bar{x}, \bar{y}) d \mid i \in \bar{I}^{G}(d)\right\}, \\
& \nabla f(\bar{x}, \bar{y})^{\top} w+d^{\top} \nabla^{2} f(\bar{x}, \bar{y}) d-\varphi^{\prime \prime}\left(\bar{x} ; \delta_{x}, \omega_{x}\right), \\
& \left.\max \left\{\nabla g_{i}(\bar{x}, \bar{y})^{\top} w+d^{\top} \nabla^{2} g_{i}(\bar{x}, \bar{y}) d \mid i \in \bar{I}^{g}(d)\right\}\right\}
\end{aligned}
$$

for all $w=\left(\omega_{x}, \omega_{y}\right) \in \mathbb{R}^{n} \times \mathbb{R}^{m}$. As a consequence, the assumptions of the proposition guarantee that

$$
\forall d \in C_{M}^{\varphi}(\bar{x}, \bar{y}) \backslash\{0\}: \quad \inf _{w \in \mathbb{R}^{n} \times \mathbb{R}^{m}} \psi^{\prime \prime}((\bar{x}, \bar{y}) ; d, w)>0
$$

holds true. Due to the above observations, Proposition 2.10 yields that the second-order growth condition holds for $\psi$ at $(\bar{x}, \bar{y})$, and due to the first part of the proof, the claim of the theorem holds. 
Let us interrelate the second-order sufficient condition of Theorem 5.3 with other second-order sufficient optimality conditions from the literature of nonlinear optimization. Therefore, we suppose for a moment that $\varphi$ is twice continuously differentiable (in particular, $-\varphi$ is second-order epi-regular in this setting). This is guaranteed under validity of LLICQ, LSOSC, and LSC at the reference point from gph $S$ provided that the lower level problem is convex for each $x \in \mathbb{R}^{n}$, and in this particular situation, the associated Hessian of $\varphi$ can be computed in terms of initial problem data, see [18, Theorem 3.4.1] for details. Exploiting the Farkas lemma, one can easily check that (5.4) equals the KKT-conditions of (LLVF) since $\varphi$ is continuously differentiable at the reference parameter. Furthermore, for fixed $d \in C_{M}^{\varphi}(\bar{x}, \bar{y}),(5.5)$ is a linear program whose dual is given by

$$
\max \left\{d^{\top} \nabla^{2} L^{\mathrm{vf}}(\bar{x}, \bar{y}, \sigma, v) d \mid(\sigma, v) \in \Lambda_{0}^{\mathrm{vf}}(\bar{x}, \bar{y}, d)\right\}
$$

where $L^{\mathrm{vf}}: \mathbb{R}^{n} \times \mathbb{R}^{m} \times \mathbb{R} \times \mathbb{R}^{p+1+q} \rightarrow \mathbb{R}$ is the generalized Lagrangian of (LLVF) given by

$$
L^{\mathrm{vf}}(x, y, \sigma, v):=\sigma F(x, y)+G(x, y)^{\top} v^{G}+v^{\mathrm{vf}}(f(x, y)-\varphi(x))+g(x, y)^{\top} v^{g}
$$

and, for each $d \in C_{M}^{\varphi}(\bar{x}, \bar{y}), \Lambda_{0}^{\mathrm{vf}}(\bar{x}, \bar{y}, d)$ is the set of Fritz-John multipliers defined below:

$$
\Lambda_{0}^{\mathrm{vf}}(\bar{x}, \bar{y}, d):=\left\{\begin{array}{l|l}
(\sigma, v) \in \mathbb{R} \times \mathbb{R}^{p+1+q} & \begin{array}{l}
\nabla L^{\mathrm{vf}}(\bar{x}, \bar{y}, \sigma, v)=0 \\
\sigma \geq 0, v \geq 0, \\
(\sigma, v) \neq 0, \\
\forall i \notin \bar{I}^{G}(d): v_{i}^{G}=0 \\
\forall i \notin \bar{I}^{g}(d): v_{i}^{g}=0
\end{array}
\end{array}\right\} .
$$

We emphasize that $\nabla L^{\mathrm{vf}}$ and $\nabla^{2} L^{\mathrm{vf}}$ denote the respective derivatives of the Lagrangian function $L^{\mathrm{vf}}$ w.r.t. the decision variables $x$ and $y$. Above and subsequently, we identify $v \in \mathbb{R}^{p+1+q}$ with the triplet $\left(v^{G}, v^{\mathrm{vf}}, v^{g}\right) \in \mathbb{R}^{p} \times \mathbb{R} \times \mathbb{R}^{q}$ where $v^{G}$ is the vector of the first $p$ components of $v, v^{\mathrm{vf}}$ is the $(p+1)$-th component of $v$ and $v^{g}$ is the vector of the last $q$ components of $v$. Using the above notation, the set

$$
\Lambda^{\mathrm{vf}}(\bar{x}, \bar{y}):=\left\{v \in \mathbb{R}^{p+1+q} \mid(1, v) \in \Lambda_{0}^{\mathrm{vf}}(\bar{x}, \bar{y}, 0)\right\}
$$

comprises the Lagrange multipliers for (LLVF) at $(\bar{x}, \bar{y})$ and is, due to (5.4), nonempty. For fixed multiplier $\bar{v} \in \Lambda^{\mathrm{vf}}(\bar{x}, \bar{y})$, we easily obtain the following representation of the critical cone from the KKT-system of (LLVF) and Remark 5.1:

$$
C_{M}^{\varphi}(\bar{x}, \bar{y})=\left\{d:=\left(\delta_{x}, \delta_{y}\right) \in \mathbb{R}^{n} \times \mathbb{R}^{m} \mid \begin{array}{rl}
\nabla G_{i}(\bar{x}, \bar{y})^{\top} d \leq 0 & i \in \bar{I}^{G}, \bar{v}_{i}^{G}=0 \\
\nabla G_{i}(\bar{x}, \bar{y})^{\top} d=0 \quad i \in \bar{I}^{G}, \bar{v}_{i}^{G}>0 \\
\nabla f(\bar{x}, \bar{y})^{\top} d-\nabla \varphi(\bar{x})^{\top} \delta_{x}= \\
\nabla g_{i}(\bar{x}, \bar{y})^{\top} d \leq 0 \quad i \in \bar{I}^{g}, \bar{v}_{i}^{g}=0 \\
\nabla g_{i}(\bar{x}, \bar{y})^{\top} d=0 \quad i \in \bar{I}^{g}, \bar{v}_{i}^{g}>0
\end{array}\right\} .
$$

Thus, for each $d \in C_{M}^{\varphi}(\bar{x}, \bar{y})$, it naturally holds $(1, \bar{v}) \in \Lambda_{0}^{\mathrm{vf}}(\bar{x}, \bar{y}, d)$, i.e. the latter set is nonempty. Coming back to Theorem 5.3, saying that (5.5) possesses positive objective value for each $d \in C_{M}^{\varphi}(\bar{x}, \bar{y}) \backslash\{0\}$ is, by strong duality of linear programming, equivalent to postulating

$$
\forall d \in C_{M}^{\varphi}(\bar{x}, \bar{y}) \backslash\{0\} \exists(\sigma, v) \in \Lambda_{0}^{\mathrm{vf}}(\bar{x}, \bar{y}, d): \quad d^{\top} \nabla^{2} L^{\mathrm{vf}}(\bar{x}, \bar{y}, \sigma, v) d>0 .
$$

Due to the above comments, this condition is implied by validity of

$$
\forall d \in C_{M}^{\varphi}(\bar{x}, \bar{y}) \backslash\{0\} \exists v \in \Lambda^{\mathrm{vf}}(\bar{x}, \bar{y}): \quad d^{\top} \nabla^{2} L^{\mathrm{vf}}(\bar{x}, \bar{y}, 1, v) d>0,
$$


and this corresponds to the classical Second-Order Sufficient Condition (SOSC) from nonlinear programming applied to (LLVF). Clearly, (5.7) is weaker than (5.8).

We observe from above that the requirements from Theorem 5.3 provide a weak Fritz-John-type second-order sufficient condition in primal form which can be dualized whenever $\varphi$ is sufficiently smooth.

Example 5.4. We consider the bilevel programming problem from Example 4.3 at $(\bar{x}, \bar{y}):=(-3,1)$. Locally around $\bar{x}, \varphi$ is affine and, thus, smooth. One can easily check that

$$
\Lambda^{\mathrm{vf}}(\bar{x}, \bar{y})=\left\{\left(v^{\mathrm{vf}}, 0, v_{2}^{g}\right) \in \mathbb{R}_{+}^{3} \mid 2-3 v^{\mathrm{vf}}+v_{2}^{g}=0\right\}
$$

holds true, and this set is nonempty, i.e. the KKT-conditions hold for the associated model (LLVF) at $(\bar{x}, \bar{y})$. Let us fix $\bar{v}^{\mathrm{vf}}:=1$ and $\bar{v}_{2}^{g}:=1$. Then, it holds $\left(\bar{v}^{\mathrm{vf}}, 0, \bar{v}_{2}^{g}\right) \in \Lambda^{\mathrm{vf}}(\bar{x}, \bar{y})$ and

$$
\nabla^{2} L^{\mathrm{vf}}\left(\bar{x}, \bar{y}, 1, \bar{v}^{\mathrm{vf}}, 0, \bar{v}_{2}^{g}\right)=\left(\begin{array}{ll}
1 & 1 \\
1 & 1
\end{array}\right) .
$$

Furthermore, one can easily check that $C_{M}^{\varphi}(\bar{x}, \bar{y})=\mathbb{R} \times\{0\}$ is valid. Consequently, condition (5.8) is satisfied, and, hence, $(\bar{x}, \bar{y})$ is a strict local minimizer of the underlying bilevel optimization problem.

Despite the above observations, Theorem 5.3 is of limited use in practice due to the following observations. First, it demands several assumptions on the implicitly given function $\varphi$. Second, the appearing linearization and critical cone depend on the directional derivative of $\varphi$. Third, the program (5.5) involves evaluations of the second-order directional derivative of the function $\varphi$. In order to obtain applicable conditions, we need to focus on specific instances of (BPP) where most of these issues can be discussed in terms of initial problem data. This will be done in the upcoming subsections for three special classes of bilevel optimization problems.

Note that (5.4) is a comparatively strong condition. Indeed, if $\varphi$ is smooth at $\bar{x}$, then this requirement is equivalent to postulating that $(\bar{x}, \bar{y})$ is a KKT-point of (LLVF), see Proposition 5.6 below. The class of bilevel programming problems whose local minimizers satisfy (abstract) KKT-conditions of (LLVF) is closely related to those programs enjoying the property to be partially calm at local minimizers.

Remark 5.5. Let all the assumptions of Theorem 5.3 be satisfied at some feasible point $(\bar{x}, \bar{y}) \in \mathbb{R}^{n} \times \mathbb{R}^{m}$ of (BPP). Thus, $(\bar{x}, \bar{y})$ is, in some sense, a stationary point of (LLVF) where a (primal) Fritz-John-type second-order sufficient optimality condition holds, cf. (5.7) for the case where $\varphi$ is twice continuously differentiable. In light of standard results regarding penalty methods, one might ask now whether $(\bar{x}, \bar{y})$ is a local minimizer of the partially penalized problem (3.3) for some $\kappa>0$ or, equivalently, whether (LLVF) is necessarily partially calm at $(\bar{x}, \bar{y})$. Following e.g. [21] or [25], this requires either some strong second-order sufficient condition to hold at $(\bar{x}, \bar{y})$ or an MFCQ-type constraint qualification to be valid for (LLVF) at $(\bar{x}, \bar{y})$. Both these requirements are, however, not reasonable in the setting at hand. We already observed that Theorem 5.3 only provides a weak second-order condition while it is folklore that (LLVF) is inherently irregular. Consequently, Theorem 5.3 might be used to identify local minimizers of (BPP) where the latter is not necessarily partially calm.

Below, we want to clarify the relationship between condition (5.4) and the property of $(\bar{x}, \bar{y})$ to be a KKT-point of (LLVF). Therefore, let us state the (nonsmooth) KKT-conditions of (LLVF) at a reference point $(\bar{x}, \bar{y}) \in M$ such that $\varphi$ is locally Lipschitzian at $\bar{x}$. We say that $(\bar{x}, \bar{y})$ is a KKT-point of (LLVF) if there are multipliers $v^{G} \in \mathbb{R}^{p}, v^{\mathrm{vf}} \geq 0$, and $v^{g} \in \mathbb{R}^{q}$ satisfying

$$
\begin{aligned}
& \nabla_{x} F(\bar{x}, \bar{y})+\nabla_{x} G(\bar{x}, \bar{y})^{\top} v^{G}+v^{\mathrm{vf}} \nabla_{x} f(\bar{x}, \bar{y})+\nabla_{x} g(\bar{x}, \bar{y})^{\top} v^{g} \in v^{\mathrm{vf}} \partial^{c} \varphi(\bar{x}), \\
& 0=\nabla_{y} F(\bar{x}, \bar{y})+\nabla_{y} G(\bar{x}, \bar{y})+v^{\mathrm{vf}} \nabla_{y} f(\bar{x}, \bar{y})+\nabla_{y} g(\bar{x}, \bar{y})^{\top} v^{g}, \\
& v^{G} \geq 0, \quad \forall i \notin \bar{I}^{G}: v_{i}^{G}=0, \\
& v^{g} \geq 0, \quad \forall i \notin \bar{I}^{g}: v_{i}^{g}=0 .
\end{aligned}
$$


Let us mention that it is not necessary to state a complementarity slackness condition w.r.t. the optimal value constraint $f(x, y)-\varphi(x) \leq 0$ and its multiplier $v^{\mathrm{vf}}$ since the constraint is active at each feasible point of (BPP). Observe that the above definition is reasonable since in case where $\varphi$ is continuously differentiable at $\bar{x}, \partial^{c} \varphi(\bar{x})$ reduces to the singleton $\{\nabla \varphi(\bar{x})\}$ and (5.9) coincides with the standard KKT-system of (LLVF).

Proposition 5.6. Let $(\bar{x}, \bar{y}) \in M$ be chosen such that $\varphi$ is directionally differentiable and locally Lipschitz continuous at $\bar{x}$. Then, the following assertions hold.

1. If (5.4) holds and if $\varphi$ is Clarke regular at $\bar{x}$, then $(\bar{x}, \bar{y})$ is a KKT-point of (LLVF).

2. If $(\bar{x}, \bar{y})$ is a KKT-point of (LLVF) and if- $\varphi$ is Clarke-regular at $\bar{x}$, then (5.4) holds.

Proof. We show both statements separately.

1. For brevity, we introduce a polyhedral cone $\mathcal{K}(\bar{x}, \bar{y}) \subseteq \mathbb{R}^{n} \times \mathbb{R}^{m}$ by

$$
\mathcal{K}(\bar{x}, \bar{y}):=\left\{\begin{array}{l|l}
d \in \mathbb{R}^{n} \times \mathbb{R}^{m} & \begin{array}{l}
\nabla G_{i}(\bar{x}, \bar{y})^{\top} d \leq 0 \\
\nabla g_{i}(\bar{x}, \bar{y})^{\top} d \leq 0 \quad i \in \bar{I}^{G} \\
g
\end{array}
\end{array}\right\} .
$$

By definition and the assumptions on $\varphi$, we have

$$
\begin{aligned}
\mathcal{L}_{M}^{\varphi}(\bar{x}, \bar{y}) & =\left\{d:=\left(\delta_{x}, \delta_{y}\right) \in \mathcal{K}(\bar{x}, \bar{y}) \mid \nabla f(\bar{x}, \bar{y})^{\top} d-\varphi^{\prime}\left(\bar{x} ; \delta_{x}\right) \leq 0\right\} \\
& =\left\{d:=\left(\delta_{x}, \delta_{y}\right) \in \mathcal{K}(\bar{x}, \bar{y}) \mid \nabla f(\bar{x}, \bar{y})^{\top} d-\varphi^{\circ}\left(\bar{x} ; \delta_{x}\right) \leq 0\right\} \\
& =\left\{d:=\left(\delta_{x}, \delta_{y}\right) \in \mathcal{K}(\bar{x}, \bar{y}) \mid \nabla f(\bar{x}, \bar{y})^{\top} d-\max \left\{\xi^{\top} \delta_{x} \mid \xi \in \partial^{c} \varphi(\bar{x})\right\} \leq 0\right\} \\
& =\bigcup_{\xi \in \partial^{c} \varphi(\bar{x})}\left\{d:=\left(\delta_{x}, \delta_{y}\right) \in \mathcal{K}(\bar{x}, \bar{y}) \mid \nabla f(\bar{x}, \bar{y})^{\top} d-\xi^{\top} \delta_{x} \leq 0\right\} .
\end{aligned}
$$

Applying the polarization rule for unions and polyhedral cones yields

$$
\begin{aligned}
\mathcal{L}_{M}^{\varphi}(\bar{x}, \bar{y})^{\circ} & =\bigcap_{\xi \in \partial^{c} \varphi(\bar{x})}\left\{d:=\left(\delta_{x}, \delta_{y}\right) \in \mathcal{K}(\bar{x}, \bar{y}) \mid \nabla f(\bar{x}, \bar{y})^{\top} d-\xi^{\top} \delta_{x} \leq 0\right\}^{\circ} \\
& =\bigcap_{\xi \in \partial^{c} \varphi(\bar{x})}\left\{\begin{array}{l}
(\eta, \theta) \\
\begin{array}{l}
\eta=\nabla_{x} G(\bar{x}, \bar{y})^{\top} v^{G}+v^{\mathrm{vf}}\left(\nabla_{x} f(\bar{x}, \bar{y})-\xi\right)+\nabla_{x} g(\bar{x}, \bar{y})^{\top} v^{g}, \\
\theta=\nabla_{y} G(\bar{x}, \bar{y})^{\top} v^{G}+v^{\mathrm{vf}} \nabla_{y} f(\bar{x}, \bar{y})+\nabla_{y} g(\bar{x}, \bar{y})^{\top} v^{g}, \\
v^{G}, v^{\mathrm{vf}}, v^{g} \geq 0, \\
\forall i \notin \bar{I}^{G}: v_{i}^{G}=0, \\
\forall i \notin \bar{I}^{g}: v_{i}^{g}=0
\end{array}
\end{array}\right\} .
\end{aligned}
$$

Now, the statement follows observing that (5.4) equals $-\nabla F(\bar{x}, \bar{y}) \in \mathcal{L}_{M}^{\varphi}(\bar{x}, \bar{y})^{\circ}$ by definition of the polar cone.

2. Let $(\bar{x}, \bar{y})$ be a KKT-point of (LLVF). Then, there are multipliers $v^{G} \in \mathbb{R}^{p}, v^{\mathrm{vf}} \geq 0$, and $v^{g} \in \mathbb{R}^{q}$ as well as $\xi \in \partial^{c} \varphi(\bar{x})$ which satisfy (5.9b), (5.9c), (5.9d), and

$$
0=\nabla_{x} F(\bar{x}, \bar{y})+\nabla_{x} G(\bar{x}, \bar{y})^{\top} v^{G}+v^{\mathrm{vf}}\left(\nabla_{x} f(\bar{x}, \bar{y})-\xi\right)+\nabla_{x} g(\bar{x}, \bar{y})^{\top} v^{g} .
$$

Now, choose $d:=\left(\delta_{x}, \delta_{y}\right) \in \mathcal{L}_{M}^{\varphi}(\bar{x}, \bar{y})$ arbitrarily. Multiplying (5.10) with $\delta_{x}$ as well as (5.9b) 
with $\delta_{y}$ and summing the resulting equations up yields

$$
\begin{aligned}
\nabla F(\bar{x}, \bar{y})^{\top} d & =-(\nabla G(\bar{x}, \bar{y}) d)^{\top} v^{G}-v^{\mathrm{vf}}\left(\nabla f(\bar{x}, \bar{y})^{\top} d-\xi^{\top} \delta_{x}\right)-(\nabla g(\bar{x}, \bar{y}) d)^{\top} v^{g} \\
& \geq v^{\mathrm{vf}}\left(\xi^{\top} \delta_{x}-\nabla f(\bar{x}, \bar{y})^{\top} d\right) \\
& \geq v^{\mathrm{vf}}\left(\min \left\{\zeta^{\top} \delta_{x} \mid \zeta \in \partial^{c} \varphi(\bar{x})\right\}-\nabla f(\bar{x}, \bar{y})^{\top} d\right) \\
& =v^{\mathrm{vf}}\left(-\max \left\{(-\zeta)^{\top} \delta_{x} \mid \zeta \in \partial^{c} \varphi(\bar{x})\right\}-\nabla f(\bar{x}, \bar{y})^{\top} d\right) \\
& =v^{\mathrm{vf}}\left(-\max \left\{\zeta^{\top} \delta_{x} \mid \zeta \in \partial^{c}(-\varphi)(\bar{x})\right\}-\nabla f(\bar{x}, \bar{y})^{\top} d\right) \\
& =v^{\mathrm{vf}}\left(-(-\varphi)^{\circ}\left(\bar{x} ; \delta_{x}\right)-\nabla f(\bar{x}, \bar{y})^{\top} d\right) \\
& =v^{\mathrm{vf}}\left(-(-\varphi)^{\prime}\left(\bar{x} ; \delta_{x}\right)-\nabla f(\bar{x}, \bar{y})^{\top} d\right) \\
& =v^{\mathrm{vf}}\left(\varphi^{\prime}\left(\bar{x} ; \delta_{x}\right)-\nabla f(\bar{x}, \bar{y})^{\top} d\right) \geq 0
\end{aligned}
$$

and this shows the desired result.

Hence, the proof is complete.

We close the abstract analysis in this section with a brief remark regarding the above result.

Remark 5.7. The proof of Proposition 5.6 even shows the following stronger statement: Fix $(\bar{x}, \bar{y}) \in M$ such that $\varphi$ is directionally differentiable, locally Lipschitz continuous, and Clarke regular at $\bar{x}$ (all these assumptions hold if $\varphi$ is locally convex around $\bar{x}$ ). If (5.4) holds, then, for each $\xi \in \partial^{c} \varphi(\bar{x})$, there exist multipliers $v^{G} \in \mathbb{R}^{p}, v^{v f} \geq 0$, and $v^{g} \in \mathbb{R}^{q}$ which satisfy (5.10), (5.9b), (5.9c), and (5.9d).

\subsection{FULLY LINEAR LOWER LEVEL}

Here, we focus on the setting

$$
\forall x \in \mathbb{R}^{n}: \quad S(x):=\underset{y}{\operatorname{argmin}}\left\{c^{\top} y \mid A x+B y \leq b\right\}
$$

where $A \in \mathbb{R}^{q \times n}, B \in \mathbb{R}^{q \times m}, b \in \mathbb{R}^{q}$, and $c \in \mathbb{R}^{m}$ are fixed matrices. Obviously, this particular lower level problem is fully linear and, thus, the associated optimal value function $\varphi$ is convex and piecewise affine on $\operatorname{dom} \varphi$. More precisely, there exist only finitely many so-called regions of stability such that $\varphi$ is affine on each of these sets. In particular, $\varphi$ can be represented as the maximum of finitely many affine functions on $\operatorname{dom} \varphi$. As a consequence, the maximum-rules from Lemmas 2.2 and 2.4 show that $\varphi$ is second-order directionally differentiable at each point $x \in \operatorname{int} \operatorname{dom} \varphi$. Clearly, $\varphi$ is Lipschitz continuous on int dom $\varphi$.

Next, we fix a point $\bar{x} \in \operatorname{dom} \varphi$. Then, $S(\bar{x}) \neq \varnothing$ holds true, and by strong duality, the solution set of the lower level dual, given by

$$
\bar{\Lambda}(\bar{x}):=\underset{\lambda}{\operatorname{argmax}}\left\{(A \bar{x}-b)^{\top} \lambda \mid B^{\top} \lambda=-c, \lambda \geq 0\right\},
$$

is nonempty as well. With the aid of this set, we can express the subdifferential of $\varphi$ at $\bar{x}$.

Lemma 5.8. Fix $\bar{x} \in \operatorname{dom} \varphi$. Then, it holds

$$
\partial \varphi(\bar{x})=\left\{A^{\top} \lambda \mid \lambda \in \bar{\Lambda}(\bar{x})\right\} .
$$

Proof. We show both inclusions separately. For the first one, fix $\xi \in \partial \varphi(\bar{x})$ and some $\bar{y} \in S(\bar{x})$. Then, it holds $c^{\top} \bar{y}=\varphi(\bar{x})$. By definition of the subdifferential (in the sense of convex analysis), $(\bar{x}, \bar{y})$ solves the linear programming problem

$$
\min _{x, y}\left\{-\xi^{\top} x+c^{\top} y \mid A x+B y \leq b\right\}
$$


i.e., we can find some $\lambda \in \mathbb{R}^{q}$ such that

$$
-\xi+A^{\top} \lambda=0, \quad c+B^{\top} \lambda=0, \quad \lambda^{\top}(A \bar{x}+B \bar{y}-b)=0, \quad \lambda \geq 0 .
$$

By strong duality of linear programming, the last three conditions ensure $\lambda \in \bar{\Lambda}(\bar{x})$.

Conversely, fix $\bar{\lambda} \in \bar{\Lambda}(\bar{x})$ and assume that $A^{\top} \bar{\lambda}$ does not belong to $\partial \varphi(\bar{x})$. Then, we find some $x \in \mathbb{R}^{n}$ such that $\varphi(x)<\varphi(\bar{x})+\bar{\lambda}^{\top} A(x-\bar{x})$ holds true. By definition of $\varphi$, there need to exist $y \in K(x)$ and $\bar{y} \in S(\bar{x})$ which satisfy $c^{\top} y<c^{\top} \bar{y}+\bar{\lambda}^{\top} A(x-\bar{x})$. Due to $\bar{\lambda} \in \bar{\Lambda}(\bar{x})$, it holds

$$
c+B^{\top} \bar{\lambda}=0, \quad \bar{\lambda}^{\top}(A \bar{x}+B \bar{y}-b)=0, \quad \bar{\lambda} \geq 0,
$$

and this yields

$$
c^{\top} y<c^{\top} \bar{y}+\bar{\lambda}^{\top} A(x-\bar{x}) \leq c^{\top} \bar{y}+\bar{\lambda}^{\top}(b-B y)+\bar{\lambda}^{\top}(B \bar{y}-b)=\left(c+B^{\top} \bar{\lambda}\right)^{\top} \bar{y}-\left(B^{\top} \bar{\lambda}\right)^{\top} y=c^{\top} y,
$$

which is a contradiction.

Now, we assume that $\bar{x} \in \operatorname{int} \operatorname{dom} \varphi$. This guarantees that $\partial \varphi(\bar{x})$ is compact. Thus, Lemma 5.8 yields

$$
\forall \delta_{x} \in \mathbb{R}^{n}: \quad \varphi^{\prime}\left(\bar{x} ; \delta_{x}\right)=\max _{\lambda \in \bar{\Lambda}(\bar{x})} \delta_{x}^{\top} A^{\top} \lambda .
$$

Noting that locally around $\bar{x}, \varphi$ equals the maximum of finitely many affine functions, we have

$$
\varphi(\bar{x}+t r)-\varphi(\bar{x})=t \varphi^{\prime}(\bar{x} ; r)
$$

for all $r \in \mathbb{R}^{n}$ provided $t>0$ is sufficiently small. Particularly, we obtain

$$
\varphi\left(\bar{x}+t \delta_{x}+\frac{1}{2} t^{2} \omega_{x}\right)-\varphi(\bar{x})=t \max _{\lambda \in \bar{\Lambda}(\bar{x})}\left\{\delta_{x}^{\top} A^{\top} \lambda+\frac{1}{2} t \omega_{x}^{\top} A^{\top} \lambda\right\}
$$

for all $\delta_{x}, \omega_{x} \in \mathbb{R}^{n}$ if only $t>0$ is sufficiently small. Noting that $A^{\top} \bar{\Lambda}(\bar{x})$ is a compact polyhedron, $\delta_{x}^{\top} A^{\top} \lambda$ dominates the maximization term as $t>0$ is small. As a consequence, for sufficiently small $t>0$, we have

$$
\varphi\left(\bar{x}+t \delta_{x}+\frac{1}{2} t^{2} \omega_{x}\right)-\varphi(\bar{x})=t \varphi^{\prime}\left(\bar{x} ; \delta_{x}\right)+\frac{1}{2} t^{2} \max _{\bar{\lambda}}\left\{\omega_{x}^{\top} A^{\top} \bar{\lambda} \mid \bar{\lambda} \in \underset{\lambda \in \bar{\Lambda}(\bar{x})}{\operatorname{argmax}} \delta_{x}^{\top} A^{\top} \lambda\right\} .
$$

As a result of these arguments, it follows

$$
\forall \delta_{x}, \omega_{x} \in \mathbb{R}^{n}: \quad \varphi^{\prime \prime}\left(\bar{x} ; \delta_{x}, \omega_{x}\right)=\max _{\bar{\lambda}}\left\{\omega_{x}^{\top} A^{\top} \bar{\lambda} \mid \bar{\lambda} \in \underset{\lambda \in \bar{\Lambda}(\bar{x})}{\operatorname{argmax}} \delta_{x}^{\top} A^{\top} \lambda\right\},
$$

and due to (5.12), $\varphi$ and $-\varphi$ both are second-order epi-regular at $\bar{x}$. The above formula for the secondorder directional derivative of $\varphi$ matches available results on the second-order directional derivative of optimal value functions in nonlinear parametric optimization, see, e.g., [41, Theorem 4.1] and [42, Theorem 4.2]. The full linearity of the lower level problem causes $\varphi$ to be a curvature-free function possessing some kinks. In the above formula, this is reflected by the fact that no second-order information on the lower level data appears since all second-order derivatives vanish.

Let us note that local minimizers of (BPP) where the lower level stage is given as in (5.11) while $G$ is affine are KKT-points of (LLVF), see [47, Corollary 4.1], and this statement is independent of the underlying upper level objective function. Even in the case where $G$ is an arbitrary smooth map, the inherent partial calmness at all local minimizers, see [48, Proposition 4.1], implies that under validity of reasonable constraint qualifications, the KKT-conditions of (LLVF) provide a necessary optimality condition for (BPP). As a consequence, there is some reasonable hope that the first-order stationarity condition (5.4) holds in the present setting although this is not naturally guaranteed if $\varphi$ is nonsmooth at the reference point, see Proposition 5.6. The above formula for the directional derivative of $\varphi$ can be used to characterize the underlying linearization cone and critical cone explicitly. Furthermore, (5.5) can be characterized in terms of initial problem data. This allows us to evaluate the second-order sufficient optimality condition from Theorem 5.3. 


\subsection{LINEAR LOWER LEVEL WITH PARAMETERS IN THE OBJECTIVE FUNCTION}

Here, we focus on the lower level problem given by

$$
\forall x \in \mathbb{R}^{n}: \quad S(x):=\underset{y}{\operatorname{argmin}}\left\{(A x+c)^{\top} y \mid B y \leq b\right\}
$$

where $A \in \mathbb{R}^{m \times n}, B \in \mathbb{R}^{q \times m}, b \in \mathbb{R}^{q}$, and $c \in \mathbb{R}^{m}$ are fixed matrices. For simplicity, let us assume that the set $K:=\left\{y \in \mathbb{R}^{m} \mid B y \leq b\right\}$ is compact. Then, dom $S=\mathbb{R}^{n}$ holds true and the associated optimal value function $\varphi: \mathbb{R}^{n} \rightarrow \mathbb{R}$ is given by

$$
\forall x \in \mathbb{R}^{n}: \quad \varphi(x)=\min \left\{(A x+c)^{\top} y_{l} \mid l=1, \ldots, \ell\right\}
$$

where $y_{1}, \ldots, y_{\ell} \in K$ are the (finitely many) vertices of $K$. Observing that $\varphi$ is the minimum of finitely many affine functions, it is globally Lipschitz continuous. Using a minimum-rule similar to the maximum-rule from Lemma 2.2, one can easily check that $\varphi$ is directionally differentiable, and it holds

$$
\forall \delta_{x} \in \mathbb{R}^{n}: \quad \varphi^{\prime}\left(\bar{x} ; \delta_{x}\right)=\min \left\{\delta_{x}^{\top} A^{\top} y_{l} \mid l \in I(\bar{x})\right\}
$$

for all $\bar{x} \in \mathbb{R}^{n}$ where we used

$$
I(\bar{x}):=\left\{\bar{l} \in\{1, \ldots, \ell\} \mid(A \bar{x}+c)^{\top} y_{\bar{l}}=\min \left\{(A \bar{x}+c)^{\top} y_{l} \mid l=1, \ldots, \ell\right\}\right\}
$$

Noting that we have $S(\bar{x})=\operatorname{conv}\left\{y_{l} \mid l \in I(\bar{x})\right\}$, it follows

$$
\forall \delta_{x} \in \mathbb{R}^{n}: \quad \varphi^{\prime}\left(\bar{x} ; \delta_{x}\right)=\min \left\{\delta_{x}^{\top} A^{\top} y \mid y \in S(\bar{x})\right\}
$$

Similar arguments as used in Section 5.1 can be exploited to infer that $\varphi$ is second-order directionally differentiable and that the formula

$$
\forall \delta_{x}, \omega_{x} \in \mathbb{R}^{n}: \quad \varphi^{\prime \prime}\left(\bar{x} ; \delta_{x}, \omega_{x}\right)=\min _{\tilde{y}}\left\{\omega_{x}^{\top} A^{\top} \tilde{y} \mid \tilde{y} \in \underset{y \in S(\bar{x})}{\operatorname{argmin}} \delta_{x}^{\top} A^{\top} y\right\}
$$

holds for all $\bar{x} \in \mathbb{R}^{n}$. Trivially, $-\varphi$ is second-order directionally differentiable, too. Noting that epi $(-\varphi)$ is a polyhedron, $-\varphi$ is second-order epi-regular, see Section 2.3.

Example 5.9. Let us consider the bilevel programming problem

$$
\min _{x, y}\left\{\frac{1}{2}(x+3)^{2}+\frac{1}{2} y^{2} \mid y \in S(x)\right\}
$$

where $S: \mathbb{R} \rightrightarrows \mathbb{R}$ is the solution map of $\min _{y}\{x y \mid y \in[0,1]\}$. Observe that this is a slightly modified version of the problem discussed in Examples 4.3 and 5.4. With the aid of Theorem 5.3, we investigate the point $(\bar{x}, \bar{y}):=(0,0)$ and note that $\varphi$ is nonsmooth there. However, the above considerations show that $\varphi$ is Lipschitzian and second-order directionally differentiable while $-\varphi$ is second-order epi-regular.

Obviously, we have $\varphi^{\prime}\left(\bar{x} ; \delta_{x}\right)=\min \left\{\delta_{x}, 0\right\}$ for all $\delta_{x} \in \mathbb{R}$. This leads to

$$
\mathcal{L}_{M}^{\varphi}(\bar{x}, \bar{y})=\mathbb{R}_{+}^{2}, \quad C_{M}^{\varphi}(\bar{x}, \bar{y})=\{0\} \times \mathbb{R}_{+} .
$$

Each nonvanishing critical direction from $C_{M}^{\varphi}(\bar{x}, \bar{y})$ is of the form $d:=\left(0, \delta_{y}\right)$ with $\delta_{y}>0$. Hence, in (5.5), $\varphi^{\prime \prime}\left(\bar{x} ; 0, \omega_{x}\right)$ needs to be evaluated, and the above formula gives us $\varphi^{\prime \prime}\left(\bar{x} ; 0, \omega_{x}\right)=\min \left\{\omega_{x}, 0\right\}$ for all $\omega_{x} \in \mathbb{R}$. Furthermore, we have $\bar{I}^{g}(d)=\varnothing$ in the situation at hand. Problem (5.5) therefore reduces to

$$
\min _{\omega_{x}, \omega_{y}, \alpha}\left\{\alpha \mid 3 \omega_{x}+\delta_{y}^{2} \leq \alpha,-\min \left\{\omega_{x}, 0\right\} \leq \alpha\right\}
$$


For $\omega_{x} \geq 0$, the first constraint implies $\alpha \geq \delta_{y}^{2}$. If $\omega_{x} \in\left(-\frac{1}{4} \delta_{y}^{2}, 0\right)$ holds, then the first constraint yields $\alpha \geq \frac{1}{4} \delta_{y}^{2}$. For $\omega_{x} \leq-\frac{1}{4} \delta_{y}^{2}$, we infer $\alpha \geq-\omega_{x} \geq \frac{1}{4} \delta_{y}^{2}$ from the second constraint. Thus, for each $d:=\left(0, \delta_{y}\right) \in C_{M}^{\varphi}(\bar{x}, \bar{y}) \backslash\{0\}$, the optimal value of the associated problem (5.5) is not smaller than $\frac{1}{4} \delta_{y}^{2}$. Consequently, Theorem 5.3 shows that $(\bar{x}, \bar{y})$ is a strict local minimizer of the given bilevel programming problem.

Let us briefly note that the first-order sufficient optimality condition from Theorem 4.2 does not hold at $(\bar{x}, \bar{y})$. This is due to the fact that the first-order derivative w.r.t. $y$ of the upper level objective function vanishes at the reference point, cf. Example 4.3 where this does not happen.

\section{$5 \cdot 3$ STABLE UNIQUE LOWER LEVEL SOLUTIONS}

In this section, we take a closer look at situations where the lower level solution under consideration is unique which is provided via a standard second-order sufficient condition and convexity. Under validity of LLICQ, one does not only obtain the continuous differentiability of $\varphi$ but also its second-order directional differentiability as well as a ready-to-use formula for the computation of the second-order directional derivative. These results are subsumed in the upcoming proposition.

Proposition 5.10. For each $x \in \mathbb{R}^{n}$, let $f(x, \cdot): \mathbb{R}^{m} \rightarrow \mathbb{R}$ be convex and let $g(x, \cdot): \mathbb{R}^{m} \rightarrow \mathbb{R}^{q}$ be componentwise convex. Fix a point $(\bar{x}, \bar{y}) \in M$ where LLICQ and LSOSC hold. Let $\bar{\lambda} \in \mathbb{R}^{q}$ be the uniquely determined associated lower level Lagrange multiplier. Then, the following assertions hold:

1. $\varphi$ is continuously differentiable at $\bar{x}$ and it holds $\nabla \varphi(\bar{x})=\nabla_{x} L(\bar{x}, \bar{y}, \bar{\lambda})$,

2. $\varphi$ is second-order directionally differentiable at $\bar{x}$ and it holds

$$
\begin{aligned}
& \varphi^{\prime \prime}\left(\bar{x} ; \delta_{x}, \omega_{x}\right)=\nabla_{x} L(\bar{x}, \bar{y}, \bar{\lambda}) \omega_{x} \\
& +\inf _{\delta_{y}}\left\{\begin{array}{l|l}
d^{\top} \nabla^{2} L(\bar{x}, \bar{y}, \bar{\lambda}) d & \begin{array}{l}
\nabla g_{i}(\bar{x}, \bar{y})^{\top} d=0 \quad i \in \bar{I}^{g}, \bar{\lambda}_{i}>0 \\
\nabla g_{i}(\bar{x}, \bar{y})^{\top} d \leq 0
\end{array} \quad i \in \bar{I}^{g}, \bar{\lambda}_{i}=0
\end{array}\right\}
\end{aligned}
$$

for all $\delta_{x}, \omega_{x} \in \mathbb{R}^{n}$ where we used $d:=\left(\delta_{x}, \delta_{y}\right) \in \mathbb{R}^{n} \times \mathbb{R}^{m}$ again,

3. $\varphi$ and $-\varphi$ are second-order epi-regular at $\bar{x}$.

Proof. Under the assumptions made, there are a neighborhood $U \subseteq \mathbb{R}^{n}$ of $\bar{x}$, a mapping $s: U \rightarrow \mathbb{R}^{m}$, and a constant $\kappa>0$ such that $S(x)=\{s(x)\}$ holds for all $x \in U$ while the upper Lipschitz property

$$
\forall x \in U: \quad\|s(x)-\bar{y}\|_{2} \leq \kappa\|x-\bar{x}\|_{2}
$$

is valid, see, e.g., [37, Section 4]. Noting that validity of LLICQ implies that the Strict MangasarianFromovitz Condition holds for the lower level problem at $(\bar{x}, \bar{y})$, the assertions of the proposition directly follow by applying [8, Theorem 4.139] as well as the subsequently stated remarks.

Remark 5.11. Suppose that all the assumptions of Proposition 5.10 are valid. Then, it holds

$$
\mathcal{L}_{M}^{\varphi}(\bar{x}, \bar{y})=\left\{d:=\left(\delta_{x}, \delta_{y}\right) \in \mathbb{R}^{n} \times \mathbb{R}^{m} \mid \begin{array}{rl}
\nabla G_{i}(\bar{x}, \bar{y})^{\top} d \leq 0 & i \in \bar{I}^{G} \\
\nabla f(\bar{x}, \bar{y})^{\top} d-\nabla_{x} L(\bar{x}, \bar{y}, \bar{\lambda})^{\top} \delta_{x} \leq 0 & \\
\nabla g_{i}(\bar{x}, \bar{y})^{\top} d \leq 0 & i \in \bar{I}^{g}
\end{array}\right\} .
$$

Since $\varphi$ is continuously differentiable, Proposition 5.6 yields that (5.4) is equivalent to the existence of multipliers $v^{G} \in \mathbb{R}^{p}, v^{\mathrm{vf}} \geq 0$, and $v^{g} \in \mathbb{R}^{q}$ which satisfy (5.9b), (5.9c), (5.9d), and

$$
0=\nabla_{x} F(\bar{x}, \bar{y})+\nabla_{x} G(\bar{x}, \bar{y})^{\top} v^{G}+\nabla_{x} g(\bar{x}, \bar{y})^{\top}\left(v^{g}-v^{\mathrm{vf}} \bar{\lambda}\right) .
$$


Let us finish this section with another illustrative example.

Example 5.12. Let us consider the bilevel programming problem

$$
\min _{x, y}\left\{(x-8)^{2}+(y-9)^{2} \mid x \geq 0, y \in S(x)\right\}
$$

where $S: \mathbb{R} \rightrightarrows \mathbb{R}$ is the solution map of $\min _{y}\left\{(y-3)^{2} \mid y^{2} \leq x\right\}$. This lower level problem is taken from [12]. One can easily check that

$$
\forall x \in \mathbb{R}: \quad S(x)=\left\{\begin{array}{ll}
\varnothing & \text { if } x<0, \\
\{\sqrt{x}\} & \text { if } 0 \leq x \leq 9, \\
\{3\} & \text { if } x>9,
\end{array} \quad \text { and } \quad \varphi(x)= \begin{cases}\infty & \text { if } x<0, \\
(\sqrt{x}-3)^{2} & \text { if } 0 \leq x \leq 9, \\
0 & \text { if } x>9 .\end{cases}\right.
$$

Consider the point $(\bar{x}, \bar{y}):=(9,3)$. For $\bar{x}$, the associated lower level problem satisfies LLICQ and LSOSC at $\bar{y}$. The uniquely determined associated Lagrange multiplier is given by $\bar{\lambda}:=0$. One obtains

$$
\mathcal{L}_{M}^{\varphi}(\bar{x}, \bar{y})=\left\{\left(\delta_{x}, \delta_{y}\right) \in \mathbb{R}^{2} \mid 6 \delta_{y}-\delta_{x} \leq 0\right\},
$$

and this leads to validity of (5.4). Furthermore, it holds

$$
C_{M}^{\varphi}(\bar{x}, \bar{y})=\left\{\left(\delta_{x}, \delta_{y}\right) \in \mathbb{R}^{2} \mid 6 \delta_{y}-\delta_{x}=0\right\} .
$$

Using formula (5.13), one obtains

$$
\forall \delta_{x}, \omega_{x} \in \mathbb{R}: \quad \varphi^{\prime \prime}\left(\bar{x} ; \delta_{x}, \omega_{x}\right)= \begin{cases}0 & \text { if } \delta_{x} \geq 0 \\ \frac{1}{18} \delta_{x}^{2} & \text { if } \delta_{x}<0 .\end{cases}
$$

Let $\left(\delta_{x}, \delta_{y}\right) \in C_{M}^{\varphi}(\bar{x}, \bar{y})$ be a nonvanishing direction. Clearly, it holds $\delta_{y}=\frac{1}{6} \delta_{x}$. Thus, (5.5) simplifies to

$$
\min _{\omega_{x}, \omega_{y}, \alpha}\left\{\alpha \mid 2 \omega_{x}-12 \omega_{y}+\left(2+\frac{1}{18}\right) \delta_{x}^{2} \leq \alpha, \frac{1}{18} \delta_{x}^{2}-\varphi^{\prime \prime}\left(\bar{x} ; \delta_{x}, \omega_{x}\right) \leq \alpha,-\omega_{x}+6 \omega_{y}+\frac{1}{18} \delta_{x}^{2} \leq \alpha\right\} .
$$

In case $\delta_{x}>0$, the second constraint yields that the minimal value of this program is bounded from below by $\frac{1}{18} \delta_{x}^{2}$. Supposing that $\delta_{x}<0$ holds, we multiply the third constraint by 2 and add it to the first one in order to see that the minimal objective value is bounded from below by $\frac{13}{18} \delta_{x}^{2}$. Consequently, Theorem 5.3 shows that $(\bar{x}, \bar{y})$ is a strict local minimizer of the given bilevel optimization problem.

\section{CONCLUSION AND PERSPECTIVES}

In this paper, we derived first- and second-order sufficient optimality conditions for optimistic bilevel optimization problems of the form (BPP). For the first-order sufficient conditions, we exploited reasonable upper estimates of the tangent cone to the bilevel feasible set. Second-order sufficient conditions were obtained using the value function reformulation (LLVF) of (BPP) as well as an appropriate second-order directional derivative. Some relations to the popular partial calmness property, which is equivalent to local exactness of the optimal value constraint as a penalty function, were discussed. In light of [25], it has to be clarified in the future which types of (strong) second-order sufficient optimality conditions addressing (BPP) already imply partial calmness at the underlying strict local minimizers, since such second-order conditions might be too selective in light of the rare number of situations where partial calmness is inherent. Observing that second-order sufficient optimality conditions are available for mathematical programs with complementarity constraints, see [40, Theorem 7], one could investigate how these conditions apply to the KKT-reformulation (KKT) of (BPP). Keeping in mind that the second-order growth condition cannot hold for (KKT) whenever there exist multiple lower level Lagrange multipliers at the reference point, see Remark 4.21, it has to be studied whether this theory can be generalized to the less restrictive situation where the mapping $\Lambda$ is allowed to be multi-valued. 


\section{REFERENCES}

[1] A. Aboussoror and S. Adly. New necessary and sufficient optimality conditions for strong bilevel programming problems. Journal of Global Optimization, 70(2):309-327, 2018.

[2] J.-P. Aubin and H. Frankowska. Set-valued Analysis. Modern Birkhäuser Classics. Birkhäuser, Boston, 2009. Reprint of the 1990 edition.

[3] J. F. Bard. Practical Bilevel Optimization: Algorithms and Applications. Kluwer Academic, Dordrecht, 1998.

[4] M. S. Bazaraa, H. D. Sherali, and C. M. Shetty. Nonlinear Programming: Theory and Algorithms. Wiley and Sons, New York, 1993.

[5] A. Ben-Tal. Second-order and related extremality conditions in nonlinear programming. Fournal of Optimization Theory and Applications, 31(2):143-165, 1980.

[6] A. Ben-Tal and J. Zowe. Necessary and sufficient optimality conditions for a class of nonsmooth minimization problems. Mathematical Programming, 24(1):70-91, 1982.

[7] J. Bonnans, R. Cominetti, and A. Shapiro. Second Order Optimality Conditions Based on Parabolic Second Order Tangent Sets. SIAM Journal on Optimization, 9(2):466-492, 1999.

[8] J. F. Bonnans and A. Shapiro. Perturbation analysis of optimization problems. Springer, New York, 2000.

[9] A. Cambini, L. Martein, and M. Vlach. Second Order Tangent Sets and Optimality Conditions. Mathematica japonicae, 49(3):451-461, 1999.

[10] F. H. Clarke. Optimization and Nonsmooth Analysis. Wiley, New York, 1983.

[11] S. Dempe. A necessary and a sufficient optimality condition for bilevel programming problems. Optimization, 25(4):341-354, 1992.

[12] S. Dempe. Foundations of Bilevel Programming. Kluwer, Dordrecht, 2002.

[13] S. Dempe and J. Dutta. Is bilevel programming a special case of a mathematical program with complementarity constraints? Mathematical Programming, 131(1):37-48, 2012.

[14] S. Dempe and N. Gadhi. Second order optimality conditions for bilevel set optimization problems. Fournal of Global Optimization, 47(2):233-245, 2010.

[15] S. Dempe, V. Kalashnikov, G. Pérez-Valdéz, and N. Kalashnykova. Bilevel Programming Problems - Theory, Algorithms and Applications to Energy Networks. Springer, Berlin, 2015.

[16] S. Dempe, V. V. Kalashnikov, and N. Kalashnykova. Optimality conditions for bilevel programming problems. In S. Dempe and V. Kalashnikov, editors, Optimization with Multivalued Mappings: Theory, Applications, and Algorithms, pages 3-28. Springer US, Boston, MA, 2006.

[17] S. Dempe and A. B. Zemkoho. The bilevel programming problem: reformulations, constraint qualifications and optimality conditions. Mathematical Programming, 138(1):447-473, 2013.

[18] A. V. Fiacco. Introduction to Sensitivity and Stability Analysis in Nonlinear Programming. Academic Press Inc., New York, 1983.

[19] M. L. Flegel and C. Kanzow. Abadie-Type Constraint Qualification for Mathematical Programs with Equilibrium Constraints. Journal of Optimization Theory and Applications, 124(3):595-614, 2005.

[2o] N. Gadhi, K. Hamdaoui, and M. El idrissi. Sufficient optimality conditions and duality results for a bilevel multiobjective optimization problem via a $\psi$ reformulation. Optimization, pages 1-22, 2019.

[21] C. Geiger and C. Kanzow. Theorie und Numerik restringierter Optimierungsaufgaben. Springer, Berlin, 2002.

[22] H. Gfrerer and D. Klatte. Lipschitz and Hölder stability of optimization problems and generalized equations. Mathematical Programming, 158(1):35-75, 2016.

[23] H. Gfrerer and B. Mordukhovich. Second-Order Variational Analysis of Parametric Constraint and Variational Systems. SIAM fournal on Optimization, 29(1):423-453, 2019.

[24] H. Gfrerer and J. V. Outrata. On Computation of Generalized Derivatives of the Normal-Cone Mapping and Their Applications. Mathematics of Operations Research, 41(4):1535-1556, 2016.

[25] S.-P. Han and O. L. Mangasarian. Exact penalty functions in nonlinear programming. Mathematical Programming, 17(1):251-269, 1979.

[26] R. Henrion and J. V. Outrata. Calmness of constraint systems with applications. Mathematical Programming, 104(2):437-464, 2005 .

[27] R. Henrion and T. Surowiec. On calmness conditions in convex bilevel programming. Applicable Analysis, 9o(6):951-970, 2011.

[28] R. Janin. Directional derivative of the marginal function in nonlinear programming. In A. V. Fiacco, editor, Sensitivity, Stability and Parametric Analysis, pages 110-126. Springer, Berlin, 1984. 
[29] J. Kyparisis. On uniqueness of Kuhn-Tucker multipliers in nonlinear programming. Mathematical Programming, 32(2):242-246, 1985.

[30] G. McCormick. Second Order Conditions for Constrained Minima. SIAM fournal on Applied Mathematics, 15(3):641-652, 1967.

[31] P. Mehlitz. On the linear independence constraint qualification in disjunctive programming. Optimization, pages $1-37,2019$.

[32] A. Mohammadi, B. S. Mordukhovich, and M. E. Sarabi. Parabolic Regularity in Geometric Variational Analysis. arXiv preprint, 2019.

[33] B. S. Mordukhovich. Variational Analysis and Applications. Springer, Berlin, 2018.

[34] J. Penot. Second-Order Conditions for Optimization Problems with Constraints. SIAM fournal on Control and Optimization, 37(1):303-318, 1998.

[35] D. Ralph and S. Dempe. Directional derivatives of the solution of a parametric nonlinear program. Mathematical Programming, 70(1):159-172, 1995.

[36] S. M. Robinson. Some continuity properties of polyhedral multifunctions. In H. König, B. Korte, and K. Ritter, editors, Mathematical Programming at Oberwolfach, pages 206-214. Springer, Berlin, 1981.

[37] S. M. Robinson. Generalized equations and their solutions, part II: Applications to nonlinear programming. In M. Guignard, editor, Optimality and Stability in Mathematical Programming, pages 200-221. Springer, Berlin, 1982.

[38] R. T. Rockafellar and R. J.-B. Wets. Variational Analysis, volume 317 of Grundlehren der mathematischen Wissenschaften. Springer, Berlin, 1998.

[39] J.-J. Rückmann and A. Shapiro. Second-order optimality conditions in generalized semi-infinite programming. Set-Valued Analysis, 9(1):169-186, 2001.

[40] S. Scheel and S. Scholtes. Mathematical Programs with Complementarity Constraints: Stationarity, Optimality, and Sensitivity. Mathematics of Operations Research, 25(1):1-22, 2000.

[41] A. Shapiro. Perturbation theory of nonlinear programs when the set of optimal solutions is not a singleton. Applied Mathematics and Optimization, 18(1):215-229, 1988.

[42] A. Shapiro. Sensitivity Analysis of Nonlinear Programs and Differentiability Properties of Metric Projections. SIAM Journal on Control and Optimization, 26(3):628-645, 1988.

[43] A. Shapiro. On concepts of directional differentiability. Fournal of Optimization Theory and Applications, 66(3):477-487, 1990

[44] K. Shimizu, Y. Ishizuka, and J. F. Bard. Nondifferentiable and two-level mathematical programming. Kluwer Academic, Dordrecht, 1997.

[45] M. Studniarski. Necessary and sufficient conditions for isolated local minima of nonsmooth functions. SIAM Journal on Control and Optimization, 24(5):1044-1049, 1986.

[46] G. Wachsmuth. On LICQ and the uniqueness of Lagrange multipliers. Operations Research Letters, 41(1):78 $-80,2013$.

[47] J. J. Ye. Nondifferentiable Multiplier Rules for Optimization and Bilevel Optimization Problems. SIAM fournal on Optimization, 15(1):252-274, 2004.

[48] J. J. Ye and D. L. Zhu. Optimality conditions for bilevel programming problems. Optimization, 33(1):9-27, 1995 .

[49] A. Zemkoho. Bilevel Programming: Reformulations, Regularity, and Stationarity. PhD thesis, Technische Universität Bergakademie Freiberg, 2012. 